\title{
A design methodology for lattice and tensegrity structures based on a stiffness and volume optimization algorithm using morphological indicators
}

International Journal of Space Structures $1-18$

(C) The Author(s) 2017

Reprints and permissions: sagepub.co.uk/journalsPermissions.nav DOI: $10.1177 / 02663511117746267$ journals.sagepub.com/home/sps (S)AGE

\author{
Pierre Latteur', Jonas Feron' and Vincent Denoel ${ }^{2}$
}

\begin{abstract}
This article presents a design methodology based on a stiffness and volume optimization algorithm for three-dimensional nonlinear hyperstatic and pre-stressed structures composed of elements only subjected to axial forces, with a special emphasis on tensegrity structures. The algorithm is based on dimensionless numbers called morphological indicators that allow finding, within a given family of structures, the geometry related to a maximum stiffness or a minimum volume of materials or the best ratio between stiffness and volume. The algorithm takes into account the buckling of the struts and different materials for cables and struts. This article first demonstrates the optimization algorithm and then gives numerical confirmations and examples.
\end{abstract}

\section{Keywords}

design methodology, lattice, mass, morphological indicators, optimization, stiffness, tensegrity, volume

\section{Introduction and historic of morphological indicators}

Tensegrity structures are part of a fascinating field of structural engineering and architecture and Skelton and De Oliveira ${ }^{1}$ point out that they could bring innovative solutions by taking inspiration from behaviors observed in the nature (deployment, control, etc.). But the fact that very few tensegrity-based civil structures have been built around the world illustrates that they are largely unknown, or at least a source of mistrust, to most practitioners, architects, and engineers. There are several reasons that can explain this fact, but among them, certainly the design and construction complexity and the nonlinear behavior which implies pre-stressing to reach the desired stiffness (Figure 1) and which can lead to an adverse effect on the volume of materials used for the structure. For tensegrity structures, the optimization of stiffness and volume is thus, more than for any other kind of structure, a key aspect.

An optimization and form-finding problem is often, for the designer, a great challenge due to the great amount of parameters that characterize a structure: the span, the width, the height, the shape, the characteristics of the cross sections, the buckling lengths, the characteristics of the materials, the loads, the pre-stress, and so on. However, optimization and form-finding algorithms can lead the designers to select the feasible ranges of tensegrity-based civil structures. Skelton and De Oliveira ${ }^{1}$ already analytically showed that some tensegrity topologies have a very efficient behavior in compression and bending. Tibert and Pellegrino $^{2}$ summarize the form-finding methods for tensegrity structures and classify them into two categories: the first one contains kinematical methods which determine the configuration of either maximal length of the struts or minimal length of the cables, while the second

\footnotetext{
'Université catholique de Louvain (UCL), Belgium

¿Université de Liège, Belgium

\section{Corresponding author:}

Pierre Latteur, Civil and Environmental Engineering, Louvain School of Engineering (EPL), Université catholique de Louvain (UCL), Place du Levant, I (Vinci), bte L5.05.0I, Louvain-la-Neuve 1348, Belgium.

Email: pierre.latteur@uclouvain.be
} 


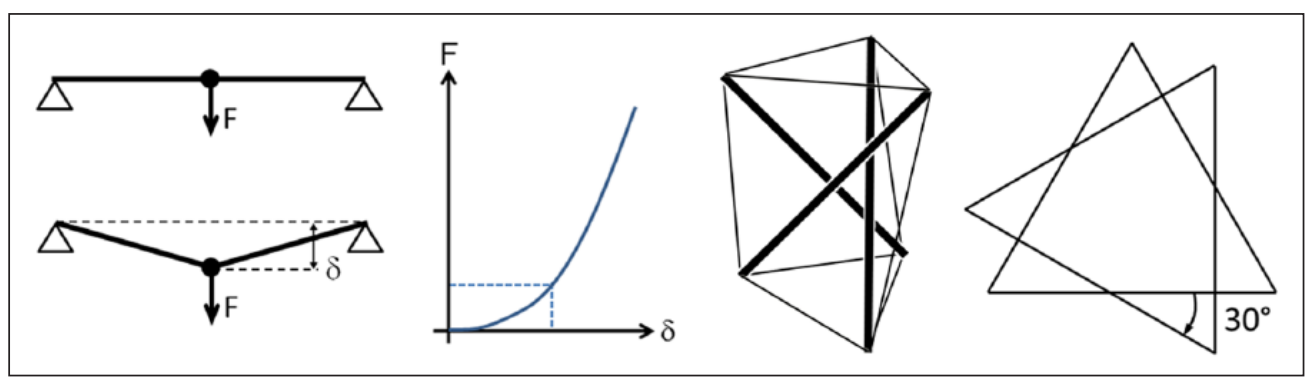

Figure I. On the left side, example of a very simple nonlinear structure, for which a classical linear approach does not give any solution because of the zero rigidity when unloaded. On the right side, the same phenomenon is observed for an elementary tensegrity modulus, unstable at first order in rotation but becoming stiffer and stiffer when the angle between both triangles deviates from the "natural" value of $30^{\circ}$.

one searches for equilibrium configurations that allow the existence of a pre-stress state with required characteristics (among them, a popular one is the force density $\operatorname{method}^{3-5}$ ). Concerning the stiffness optimization (or stiffness-to-mass (or volume) optimization), several authors added significant contribution. De Jager and Skelton ${ }^{6}$ developed a numerical method to find the geometries of planar tensegrity structures with optimal stiffness or stiffness-to-mass properties and to offer guidelines in the design. Masic et al. ${ }^{7}$ developed a very efficient numerical algorithm that allows finding the topology, geometry, and pre-stress of a structure that yields optimal design for different scenarios. The algorithm takes into account the buckling of the struts and three-dimensional (3D) tensegrity structures. Starting from an initial layout, it allows determining the best node positions, best number of stages, and best geometrical ratio. In this sense, this study is thus significant and is likely to provide a very useful tool for the designers.

Considering the design of pedestrian bridges composed of tensegrity modulus in particular, RhodeBarbarigos et al. ${ }^{8}$ propose a design method which allows finding the optimum section sizes considering the selfweight, a limited deflection, and the buckling of the struts, for a structure defined by its topology, span, height, materials, loads, pre-stress, and number of modulus. The structural performances, such as the displacements, are then evaluated through parametric studies of the topology of modulus, the level of pre-stress, and the materials used for cables and struts. In the same way, Bel Hadj Ali et al. ${ }^{9}$ study the design of pedestrian bridges considering the dynamical behavior of a defined structure. The natural frequencies are then evaluated through parametric studies of the pre-stress level and cross section of struts and cables.

This article also focuses on the designer's point of view by providing him with an "as simple as possible" design methodology, guided by the wish of simplifying the optimization and the design process by reducing the amount of parameters, by grouping them into dimensionless numbers called morphological indicators.
Their first traces appear in 1980, when Zalewski, ${ }^{10}$ a professor at the Massachusetts Institute of Technology, writes notes for his students in architecture. In his study, Zalewski compares the weight and the stiffness of various types of two-dimensional (2D) trusses whose morphology is inspired, on one hand, by the observation of flow constraints in the beams and, on the other hand, by Michell ${ }^{11}$ studies in 1904. A significant extension of Michell's theory was done by Skelton and De Oliveira ${ }^{12}$ in 2010. In his document, Zalewski already shows the relationship between the volume and deflection of simple structures and their geometric slenderness $L / H$ ( $L$ and $H$ being, respectively, the span and the height of the structure). Zalewski and Kus ${ }^{13}$ have summarized their studies in a publication presented at an IASS congress in 1996.

Then, Quintas Ripoll ${ }^{14,15}$ publishes in 1989 and 1992 two articles about the optimization of simple lattices and bows, also highlighting the direct link between their volume and their geometric slenderness $L / H$.

In 1997, Samyn compares a large amount of structures and also shows that their self-weight can be studied through a dimensionless number that he calls the indicator of volume. All his publications, including those concerning his $\mathrm{PhD}$ thesis submitted in $2000,{ }^{16}$ are summarized in a book published in 2004 in the class of the Belgian Royal Academy of Sciences. ${ }^{17}$

From 1998 on, Latteur extends the applications of the indicators of volume and displacement by developing the concept of buckling indicator and efficiency curve. The scope of the theory is then extended to 3D structures subjected to buckling and random load cases. ${ }^{18} \mathrm{He}$ also highlights new indicators, such as the self-weight indicator $\Phi=\rho L / \sigma$, the rotation indicator $\Theta=E \theta / \sigma$ and the bending indicator $Z=A h^{2} / I$.

In his thesis presented in 2006, Van Steirteghem ${ }^{19}$ has extended the theory by developing a first frequency indicator, which allowed to significantly expand fields of application of this theory to dynamically loaded structures.

In 2010, the PhD thesis of Vandenbergh ${ }^{20}$ extends the application field of morphological indicators by considering 


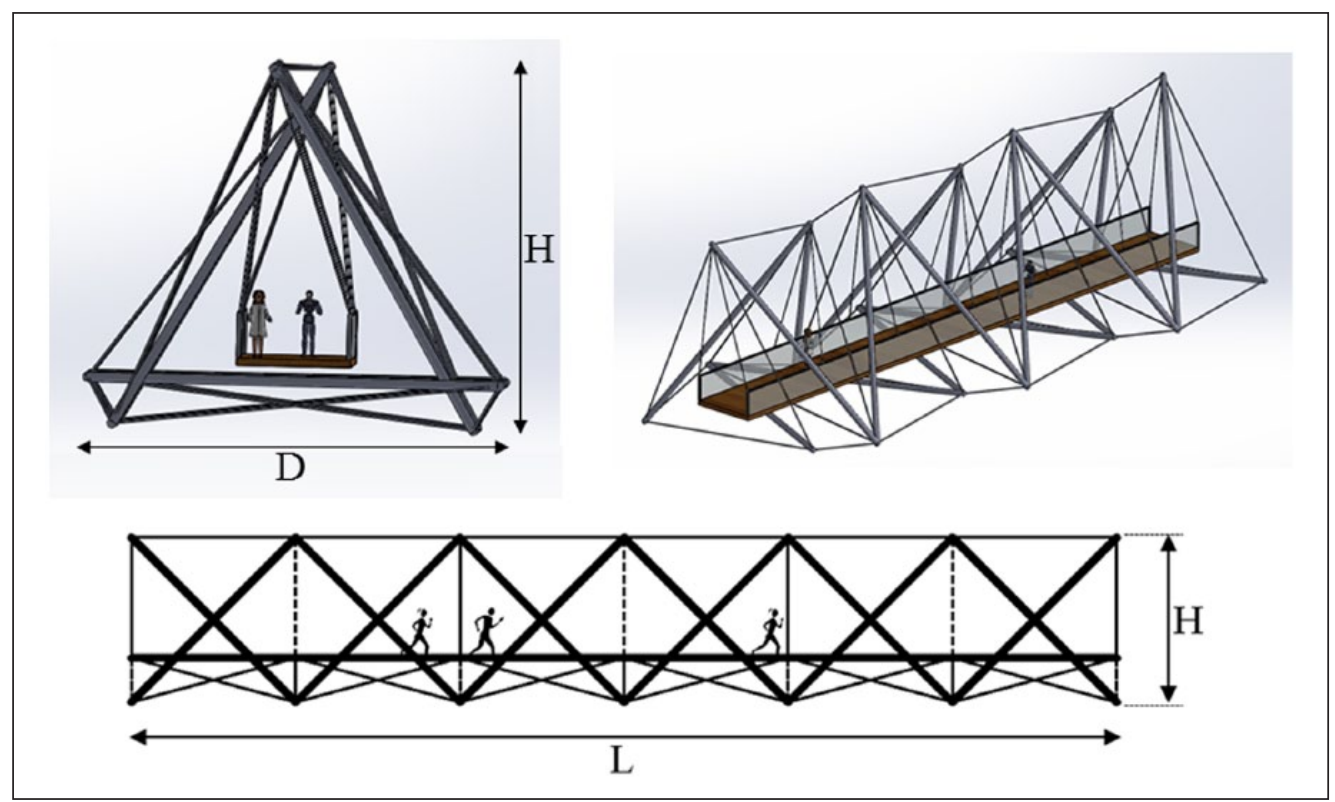

Figure 2. Example of a footbridge composed of a succession of elementary tensegrity modulus (called here "Simplex"), with a deck suspended to upper nodes.

quasi-static vibrations and global in-plane instabilities, on top of the traditional approach based on strength and local buckling.

All these researches, summarized by Vandenbergh and De Wilde, ${ }^{21}$ concern linear isostatic structures and mostly 2D trusses and arches.

The aim of this article is to extend the theory of morphological indicators to structures combining a nonlinear behavior, hyperstatic conditions, and pre-stressing, which is particularly the case for tensegrity structures.

\section{Assumptions[AQ: 1]}

We consider any structure:

- Of span $L$, height $H$, and width $D$, rigorously, considered after application of pre-stress (see Figure 2);

- Subjected to a particular external load $\tilde{F}$ acting on each of the $\mathrm{n}$ nodes according to the three directions $(X, Y, Z)$, such as

$$
\begin{aligned}
\tilde{F} & =\tilde{f} * F=\left(F_{1, X}, F_{1, Y}, F_{1, Z}, \ldots, F_{Z, 3 n}\right) \\
& =\left(t_{1}^{F}, t_{2}^{F}, t_{3}^{F}, \ldots t_{3 n}^{F}\right) * F
\end{aligned}
$$

with $-1 \leq t_{i}^{F} \leq 1$ and $\sum\left|t_{i}^{F}\right|=1$

- Where each element $i$ is, before application of $\tilde{F}$, subjected to an axial force $P_{i}$ due to the pre-stress (details in section "Pre-stress"). However, no prestress is a particular case for which the design methodology is also applicable;
- With a nonlinear behavior, although the linear behavior is a particular case for which the design methodology is also applicable;

- With supports anywhere and of any degree of redundancy;

- With cables made out of a same material of Young's modulus $E_{c}$ and strength limit $\sigma_{c}$;

- With struts made out of a same material of Young's modulus $E_{s}$ and strength limit $\sigma_{s}$ such that factor $u$ is defined as $u=\sigma_{c} / \sigma_{s}$;

- With struts with a cross-sectional $A_{s}$ and a moment of inertia $I_{s}$, such that the form factor $q$, defined as $q=I_{s} / A_{s}^{2}$, is supposed to be equal for all struts;

- Related to an indicator of buckling $\Psi$ developed by Latteur ${ }^{18}$ and such that $\Psi=\sigma_{s} L / \sqrt{q E_{s} F}$;

- With a maximum deflection $\delta$ somewhere, for instance, at mid-span and vertically;

- With a total volume $V$ of materials (cables and struts).

In this article, we use the following definitions:

- The pre-stress state $\tilde{p}$ is the elementary repartition of the axial forces in each element (with tension for cables and compression for struts), before application of the external load $\tilde{F}$;

- The pre-stress level $\beta$ allows to multiply the values of $\tilde{p}$ by a factor $\beta F$;

- The pre-stress scenario $\tilde{P}$ is the multiplication of $\tilde{p}$ and $\beta F$; 
- A self-stress mode is a particular value of $\tilde{p}$ which maintains the initial geometry of the structure;

- A self-stress state is either a self-stress mode or the combination of several self-stress modes.

\section{Other assumptions}

- $L_{0}$ is the length of any element after application of pre-stress $\tilde{P}$.

- $L_{d e f}$ is the length of any element after application of the external load $\tilde{F}$.

- Displacements $d_{i}$ and $\delta$ are the ones created by the external load $\tilde{F}$ only, and not the pre-stress.

- The materials have an elastic behavior with a ratio $\left|L_{d e f}-L_{i n i}\right| / L_{i n i}$ limited to 0.01 , which also means that $\left|L_{d e f}-L_{0}\right| / L_{0} \leq 0.01 \quad$ and $\left|L_{0}-L_{i n i}\right| / L_{i n i} \leq 0.01 \quad$ or $\quad 0.99 \leq\left|L_{d e f} / L_{0}\right| \leq 1.01$ and $0.99 \leq\left|L_{0} / L_{i n i}\right| \leq 1.01$. For steel, that means a stress that reaches $2100 \mathrm{MPa}$, which is only possible for cables with an extremely high strength limit. For usual steel and other materials, the ratio seldom exceeds 0.002 .

- For a cable (or any element remaining always in tension) of index $i$, the design criterion is [AQ: 2]

$$
\frac{N_{c, i}}{A_{c, i}}=\sigma_{c}
$$

For a strut of index $i$, the design criterion is the following approximation of corrected Euler's law

$$
\frac{N_{s, i}}{A_{s, i}}=\frac{1}{1+\Lambda_{i}^{2}} \sigma_{s} \text { with }\left\{\begin{array}{l}
\Lambda_{i}=\frac{\lambda_{i}}{\left(\pi \sqrt{\frac{E_{s}}{\sigma_{s}}}\right)} \\
\lambda_{i}=L_{0} \sqrt{\frac{A_{s, i}}{I_{s, i}}}
\end{array}\right.
$$

In section "Generalization for struts with different crosssectional areas," it is proven that the design methodology is valid both for a situation where each single element is designed according to equations (1a) and (1b), which lead to a fully stressed design, and for a situation where each cable or strut has the same section, respectively, equal to the most solicited cable or strut.

- Self-weight acting as a load case is neglected, in order to lighten the demonstration. However, it has been proved that it can be taken into account via the selfweight indicator $\Phi=\rho L / \sigma$ developed by Latteur. ${ }^{18}$

- In the same way, random external loads are not taken into account. Note that Latteur ${ }^{18}$ includes a large discussion about random loads for structures with a linear behavior.

\section{Pre-stress}

A tensegrity structure would not maintain its initial shape until appropriate pre-stress, called a self-stress state, is assigned. For a tensegrity structure with given shape, the choice of the self-stress state that leads to the maximum stiffness is itself a complex optimization problem which has been studied by Zhang and Feng ${ }^{22}$ with, furthermore, a very well-developed literature survey over this subject. This article first proposes two methods to compute the independent self-stress mode(s) of symmetric tensegrity structures. Then, different algorithms are presented to determine the self-stress state, by cleverly combining these self-stress modes, which maximize the global stiffness of the structure on the basis of a same pre-stress level.

The self-stress state could also been optimized with regard to the behavior of a dynamically loaded tensegrity structure. Ashwear et al. ${ }^{23}$ propose a method leading to an optimum self-stress state with relatively high stiffness of the structure as well as a lowest natural frequency as high as possible.

A judicious choice of pre-stress scenario $\tilde{P}$ is necessary, to ensure the stability of the tensegrity structure, to prevent some cables from slack when the external load case $\tilde{F}$ is applied and, finally, to reach the desired stiffness. The term "tensegrity structure" is, in this article, referring to a pin-jointed cables-struts assembly subjected to any pre-stress scenario $\tilde{P}$, which may not be related to a self-stress state.

Vector $\tilde{P}$ is composed of the $n_{c}+n_{s}$ values of the axial force $P_{i}$ in each element, cable or strut (with tension for cables and compression for struts). Those values are thus the internal axial forces in the elements that exist before the application of the external load $\tilde{F}$. In this article, the pre-stress scenario $\tilde{P}$ is defined as follows

$$
\tilde{P}=\tilde{p}(\beta F)=\left(t_{1}^{P}, t_{2}^{P}, \ldots, t_{i}^{P}, \ldots, t_{n_{c}+n_{s}}^{P}\right)(\beta F)
$$

where $\left\{\begin{array}{l}-1 \leq \tilde{p}=\left(t_{1}^{P}, t_{2}^{P}, \ldots, t_{i}^{P}, \ldots, t_{n_{c}+n_{s}}^{P}\right) \leq 1 \\ : \text { pre-stress state } \\ \beta \geq 0: \text { pre-stress level }\end{array}\right.$

For a pre-stress state $\tilde{p}$ judiciously chosen, $\beta_{\text {min }}$ is defined as being the particular value of $\beta$ that leads to a situation where the axial force in the least tensioned cable after application of the external load $\tilde{F}$ is equal to zero, which means that no cable slacks

$$
\beta=\theta \beta_{\min } \quad \text { with } \theta \geq 1
$$

In this article, $\theta=1$ and $\beta=\beta_{\text {min }}$ will be assumed and the way to find the value $\beta_{\min }$ is discussed in section "Discussion about the value of $\beta$." Note that one could eventually choose $\theta>1$, which is a way to improve the stiffness but which impacts $V$. 
Practically, pre-stress can be set into a tensegrity structure by introducing traction into the cables or/and compression into the struts, for instance, by placing a mechanical device at one of their extremity. The system will be such that it will shorten a cable or lengthen a strut.

There exist several pre-stress scenarios $\tilde{P}$ that guaranty that no cable will slack when the external load $\tilde{F}$ is applied. For instance, one potentially possible scenario could be coming from an elongation of each strut generated by its associated mechanical device. Therefore, one of these possible pre-stress scenarios could be relative to a pre-stress state $\tilde{p}$ which is not a self-stress state.

Section "Choice of a pre-stress scenario $\tilde{P}$ " discusses in detail the assumptions made in order to make the design methodology relevant despite the fact that the choice of the pre-stress state $\tilde{p}$ influences the stiffness and the volume.

\section{Aim and structure of this article}

Figure 2 shows an example of tensegrity footbridge composed of a number $S=6$ Simplex modulus, in which the deck is suspended to upper nodes. The following questions could be asked: for a given span $L$ and a given external load case $\tilde{F}$ , what are the values of $S$ and of the height $H$ that minimize the deflection $\delta$ at mid-span or the total volume $V$ of materials, under constraints (1a) and (1b)? And how to find a prestress scenario $\tilde{P}$ and a pre-stress state $\tilde{p}$ compatible with the external load $\tilde{F}$ ? Being able to answer to these questions via a simple methodology is the aim of this article.

Practically, this article aims at proving that for a given family of structures, $f$ being any function:

- The deflection $\delta / L$ only depends on seven dimensionless numbers, according to

$$
\frac{\delta}{L}=f\left(\frac{L}{H}, \frac{L}{D}, \Psi, \frac{E_{c}}{\sigma_{c}}, \frac{E_{s}}{\sigma_{s}}, S, \tilde{p}\right)
$$

- The volume $V$ of materials, defined by its indicator of volume $W$, only depends on the same seven dimensionless numbers and $u=\sigma_{c} / \sigma_{s}$, according to

$$
W=\frac{\sigma_{s} V}{F L}=f\left(\frac{L}{H}, \frac{L}{D}, \Psi, \frac{E_{c}}{\sigma_{c}}, \frac{E_{s}}{\sigma_{s}}, S, \tilde{p}, u\right)
$$

The demonstration is analytically developed in sections "Demonstration of relation $\delta / L=f\left(L / H, L / D, \Psi, E_{c} / \sigma_{c}, E_{s} / \sigma_{s}, \tilde{p}\right)$ for a given $S$ " and "Demonstration of relation $\sigma_{s} V / F L=f\left(L / H, L / D, \Psi, E_{c} / \sigma_{c}, E_{s} / \sigma_{s}, \tilde{p}, u\right)$ for a given $S$ " and then confirmed numerically with examples in section "Numerical confirmation of equations (28) and

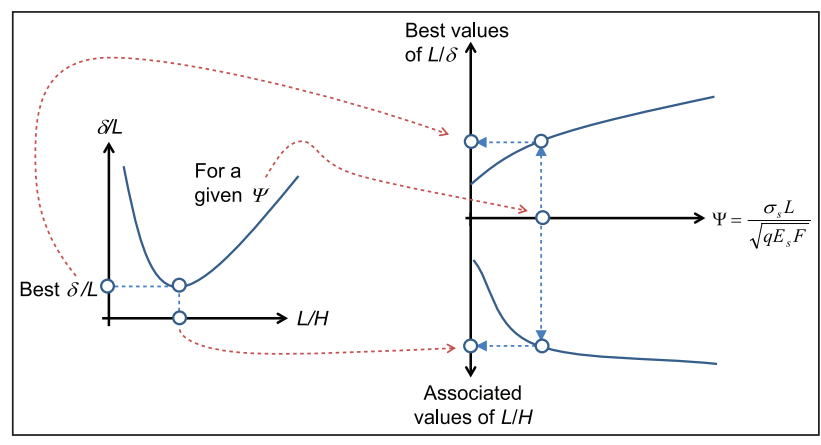

Figure 3. Curves of efficiency (see sections "Numerical confirmation of equations (28) and (29)," "Example of curves of efficiency," and "Other examples: trusses and other tensegrity topologies" for details and comments).

(29)." The way to find $\tilde{p}$ is detailed in sections "Choice of a pre-stress scenario $\tilde{P}$ " and "Optimization algorithm."

Assuming that equations (2a) and (2b) are correct, they allow to easily find the stiffest or the lightest structure, thanks to the curves shown in Figure 3. Indeed, assuming that

- A pre-stress state $\tilde{p}$ can be found and depends itself on parameters $\left(L / H, L / D, \Psi, E_{c} / \sigma_{c}, E_{s} / \sigma_{s}, S\right)$, which is justified in section "Choice of a pre-stress scenario $\tilde{P}$ ";

- The materials are chosen $\left(E_{c} / \sigma_{c}, E_{s} / \sigma_{s}\right.$, and $u$ fixed);

- $D$ is proportional to $H$ for a given family of structures;

- Relations (2a) and (2b) become for a given number $S$ of elementary modulus

$$
\begin{aligned}
& \frac{\delta}{L}=f\left(\frac{L}{H}, \Psi\right) \\
& \frac{\sigma_{s} V}{F L}=f\left(\frac{L}{H}, \Psi\right)
\end{aligned}
$$

Using relations (3a) and (3b), the optimization and the design process are thus greatly simplified, as the deflection $\delta / L$ and the indicator of volume $W$ only depend on the two parameters $L / H$ and $\Psi$. This is illustrated in Figure 3 for the deflection.

The algorithm used to draw Figure 3 is described in section "Optimization algorithm" and numerical examples are then given in sections "Numerical confirmation of equations (28) and (29)," "Example of curves of efficiency," and "Other examples: trusses and other tensegrity topologies." The left of Figure 3 shows that for a given value of the buckling indicator $\Psi$, the minimum value of $\delta / L$ is numerically found, and the corresponding values $(\Psi, L / H$, $L / \delta$ ) are reported on the right of Figure 3, called curve of efficiency. ${ }^{18}$ This process is numerically repeated for values of $\Psi$ from 0 to 100 . For a given practical case related 


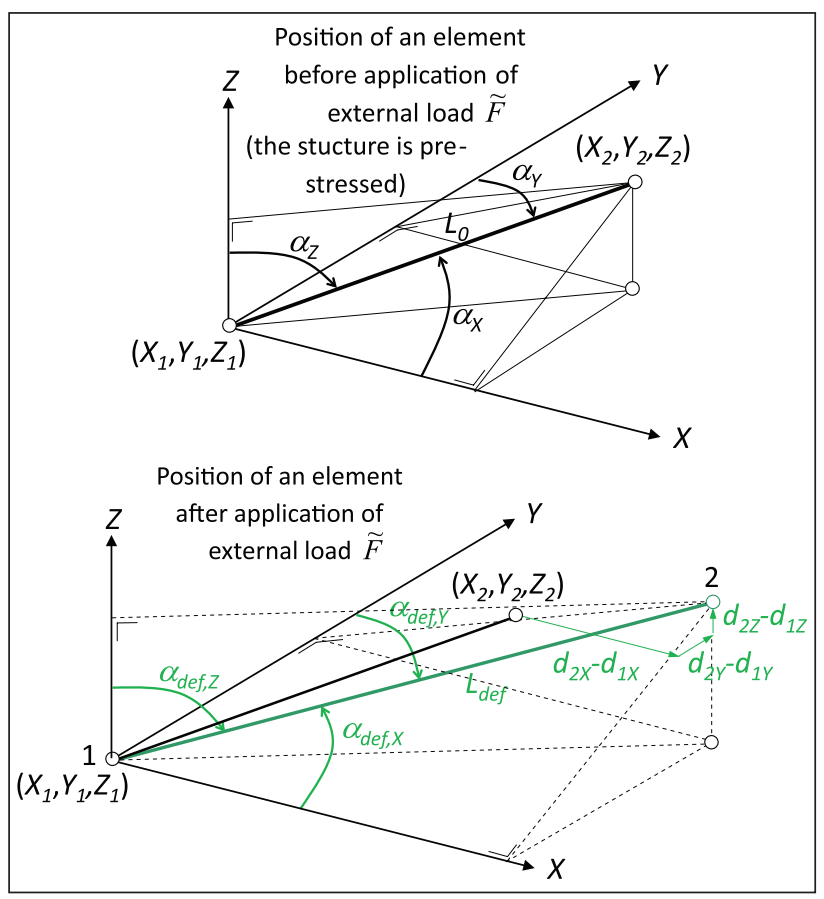

Figure 4. Node I was drawn at the same place in both configurations (before and after application of external load $\tilde{F}$ ) for the purpose of the demonstration.

to a given value of $\Psi$, the efficiency curve gives the best value $L / H$, and the associated (best) value $\delta / L$ (or $L / \delta$ ).

\section{Demonstration of relation $\delta / L=f\left(L / H, L / D, \Psi, E_{c} / \sigma_{c}, E_{s} / \sigma_{s}, \tilde{p}\right)$ for a given $S$}

Figure 4 shows that before application of $\tilde{F}$

$$
\left\{\begin{array}{l}
c_{X}=\cos \alpha_{X}=\frac{\left(X_{2}-X_{1}\right)}{L_{0}}=a\left(\frac{L}{L_{0}}\right) \\
c_{Y}=\cos \alpha_{Y}=\frac{\left(Y_{2}-Y_{1}\right)}{L_{0}}=b\left(\frac{D}{L_{0}}\right) \\
c_{Z}=\cos \alpha_{Z}=\frac{\left(Z_{2}-Z_{1}\right)}{L_{0}}=c\left(\frac{H}{L_{0}}\right)
\end{array}\right.
$$

And after application of $\tilde{F}$

$$
\left\{\begin{array}{l}
c_{d e f, X}=\cos \alpha_{d e f, X}=\frac{\left(\left(X_{2}-X_{1}\right)+\left(d_{2 X}-d_{1 X}\right)\right)}{L_{d e f}} \\
c_{d e f, Y}=\cos \alpha_{d e f, Y}=\frac{\left(\left(Y_{2}-Y_{1}\right)+\left(d_{2 Y}-d_{1 Y}\right)\right)}{L_{d e f}} \\
c_{d e f, Z}=\cos \alpha_{d e f, Z}=\frac{\left(\left(Z_{2}-Z_{1}\right)+\left(d_{2 Z}-d_{1 Z}\right)\right)}{L_{d e f}}
\end{array}\right.
$$

\section{Dimensionless expression of $L_{0} / L$}

The length $L_{0}$ of any element before application of $\tilde{F}$ is given by

$$
\begin{aligned}
L_{0} & =\sqrt{\left(X_{2}-X_{1}\right)^{2}+\left(Y_{2}-Y_{1}\right)^{2}+\left(Z_{2}-Z_{1}\right)^{2}} \\
& =\sqrt{a^{2} L^{2}+b^{2} D^{2}+c^{2} H^{2}}
\end{aligned}
$$

which leads to

$$
\frac{L_{0}}{L}=f\left(\frac{L}{H}, \frac{L}{D}\right)
$$

Dimensionless expression of $L_{\text {def }} / L$

$$
L_{\text {def }}=\sqrt{\begin{array}{l}
\left(\left(X_{2}-X_{1}\right)+\left(d_{2 X}-d_{1 X}\right)\right)^{2}+ \\
\left(\left(Y_{2}-Y_{1}\right)+\left(d_{2 Y}-d_{1 Y}\right)\right)^{2}+ \\
\left(\left(Z_{2}-Z_{1}\right)+\left(d_{2 Z}-d_{1 Z}\right)\right)^{2}
\end{array}}
$$

which gives

$$
\begin{aligned}
L_{d e f}^{2}= & L_{0}^{2}+\left[2\left(X_{2}-X_{1}\right)\left(d_{2 X}-d_{1 X}\right)+\left(d_{2 X}-d_{1 X}\right)^{2}\right] \\
& +\left[2\left(Y_{2}-Y_{1}\right)\left(d_{2 Y}-d_{1 Y}\right)+\left(d_{2 Y}-d_{1 Y}\right)^{2}\right] \\
& +\left[2\left(Z_{2}-Z_{1}\right)\left(d_{2 Z}-d_{1 Z}\right)+\left(d_{2 Z}-d_{1 Z}\right)^{2}\right]
\end{aligned}
$$

According to the assumption that $0.99<L_{d e f} / L_{0}<1.01$ (section "Assumptions"), one obtains

$$
\begin{aligned}
L_{d e f}^{2}-L_{0}^{2} & =2 L_{0}\left(L_{d e f}-L_{0}\right) * \frac{\left(L_{d e f}+L_{0}\right)}{2 L_{0}} \\
& \cong 2 L_{0}\left(L_{\text {def }}-L_{0}\right)
\end{aligned}
$$

Eliminating the term $L_{d e f}^{2}-L_{0}^{2}$ by combining equations (7) and (8), one obtains

$$
L_{d e f}-L_{0}=\left[\begin{array}{c}
c_{X}\left(d_{2 X}-d_{1 X}\right)+c_{Y}\left(d_{2 Y}-d_{1 Y}\right) \\
+c_{Z}\left(d_{2 Z}-d_{1 Z}\right) \\
\left(d_{2 X}-d_{1 X}\right)^{2}+\left(d_{2 Y}-d_{1 Y}\right)^{2} \\
+\frac{+\left(d_{2 Z}-d_{1 Z}\right)^{2}}{2 L_{0}}
\end{array}\right]
$$




$$
\frac{L_{d e f}}{L}=\frac{L_{0}}{L}+\left[\begin{array}{l}
c_{X} \frac{\left(d_{2 X}-d_{1 X}\right)}{L}+c_{Y} \frac{\left(d_{2 Y}-d_{1 Y}\right)}{L} \\
+c_{Z} \frac{\left(d_{2 Z}-d_{1 Z}\right)}{L} \\
\\
\left(d_{2 X}-d_{1 X}\right)^{2}+\left(d_{2 Y}-d_{1 Y}\right)^{2} \\
+\frac{+\left(d_{2 Z}-d_{1 Z}\right)^{2}}{2 L L_{0}}
\end{array}\right]
$$

And according to equation (6)

$$
\frac{L_{d e f}}{L}=f\left(\frac{L}{H}, \frac{L}{D}\right)+\left[\begin{array}{c}
c_{X} \frac{\left(d_{2 X}-d_{1 X}\right)}{L}+c_{Y} \frac{\left(d_{2 Y}-d_{1 Y}\right)}{L} \\
+c_{Z} \frac{\left(d_{2 Z}-d_{1 Z}\right)}{L} \\
+0.5 f^{-1}\left(\frac{L}{H}, \frac{L}{D}\right)\left(\begin{array}{l}
\left(\frac{d_{2 X}-d_{1 X}}{L}\right)^{2} \\
+\left(\frac{d_{2 Y}-d_{1 Y}}{L}\right)^{2} \\
+\left(\frac{d_{2 Z}-d_{1 Z}}{L}\right)^{2}
\end{array}\right)
\end{array}\right]
$$

And finally

$$
\frac{L_{d e f}}{L}=f\left(\frac{L}{H}, \frac{L}{D}, \ldots, \frac{d_{i}}{L}, \ldots\right)
$$

where $f$ is another function, different from the one in equation (6).

\section{Axial force $N$ in any element after application of external load $\tilde{F}$}

Before application of the external load $\tilde{F}$, each element is subjected to a pre-stress $P_{i}=\beta F t_{i}^{P}$, which is thus a particular value of vector $\tilde{P}=\tilde{p} \beta F=\left(t_{1}^{P}, t_{2}^{P}, t_{3}^{P}, \ldots, t_{n_{c}+n_{s}}^{P}\right) * \beta * F . L_{0}$ and $L_{\text {def }}$ being, respectively, the length of the element $i$ before and after application of external load $\tilde{F}$, and the axial force in the element subjected to both $\tilde{P}$ and $\tilde{F}$ is equal to

$$
N=E A \frac{\left(L_{d e f}-L_{0}\right)}{L_{0}}+P_{i}
$$

$N$ can also be written as $N=E A\left(\left(L_{d e f} / L-L_{0} / L\right) / L_{0} / L\right)+\beta F t_{i}^{P}$ or, according to equations (6) and (10), under the generic form

$$
N=E A * f\left(\frac{L}{H}, \frac{L}{D}, \ldots, \frac{d_{i}}{L}, \ldots\right)+\beta F t_{i}^{P}
$$

\section{Stiffness matrix of a deformed element in the global frame $(X, Y, Z)$}

One considers here an element $i$ (strut or cable) after application of the pre-stress $\tilde{P}$ and the external load $\tilde{F}$ and subjected to an axial force $N$. The three components of $N$ at node 1 of the element $i$, according to the three directions $(X, Y, Z)$ of the global axis system, are as follows

$$
\left\{\begin{aligned}
N_{1 X} & =-N \cos \alpha_{\text {def }, X} \\
& =-N \frac{\left(\left(X_{2}-X_{1}\right)+\left(d_{2 X}-d_{1 X}\right)\right)}{L_{d e f}} \\
N_{1 Y} & =-N \cos \alpha_{d e f, Y} \\
& =-N \frac{\left(\left(Y_{2}-Y_{1}\right)+\left(d_{2 Y}-d_{1 Y}\right)\right)}{L_{d e f}} \\
N_{1 Z} & =-N \cos \alpha_{d e f, Z} \\
& =-N \frac{\left(\left(Z_{2}-Z_{1}\right)+\left(d_{2 Z}-d_{1 Z}\right)\right)}{L_{d e f}}
\end{aligned}\right.
$$

And for node 2

$$
\left\{\begin{array}{l}
N_{2 X}=N \cos \alpha_{d e f, X}=N \frac{\left(\left(X_{2}-X_{1}\right)+\left(d_{2 X}-d_{1 X}\right)\right)}{L_{d e f}} \\
N_{2 Y}=N \cos \alpha_{d e f, Y}=N \frac{\left(\left(Y_{2}-Y_{1}\right)+\left(d_{2 Y}-d_{1 Y}\right)\right)}{L_{d e f}} \\
N_{2 Z}=N \cos \alpha_{d e f, Z}=N \frac{\left(\left(Z_{2}-Z_{1}\right)+\left(d_{2 Z}-d_{1 Z}\right)\right)}{L_{d e f}}
\end{array}\right.
$$

Assuming that $\left|L_{0} / L_{\text {def }}\right| \approx 1$ (section "Other assumptions"), equations (12) and (13) can be written according to the following matrix form that, if developed, is composed of an elastic term and a geometric term 


$$
\begin{aligned}
\left(\begin{array}{l}
N_{1 X} \\
N_{1 Y} \\
N_{1 Z} \\
N_{2 X} \\
N_{2 Y} \\
N_{2 Z}
\end{array}\right)= & \left(\begin{array}{c}
-c_{X} \\
-c_{Y} \\
-c_{Z} \\
c_{X} \\
c_{Y} \\
c_{Z}
\end{array}\right) \\
& +\frac{N}{L_{0}}\left(\begin{array}{cccccc}
1 & 0 & 0 & -1 & 0 & 0 \\
0 & 1 & 0 & 0 & -1 & 0 \\
0 & 0 & 1 & 0 & 0 & -1 \\
-1 & 0 & 0 & 1 & 0 & 0 \\
0 & -1 & 0 & 0 & 1 & 0 \\
0 & 0 & -1 & 0 & 0 & 1
\end{array}\right)\left(\begin{array}{c}
d_{1 X} \\
d_{1 Y} \\
d_{1 Z} \\
d_{2 X} \\
d_{2 Y} \\
d_{2 Z}
\end{array}\right)
\end{aligned}
$$

\section{Expression of $d_{i} / L$, for a given $S$}

Let us consider the first equation of (12). According to equations (10) and (4), it can be written according to the following generic form

$$
N_{1 X}=N * f\left(\frac{L}{H}, \frac{L}{D}, \ldots, \frac{d_{i}}{L}, \ldots\right)
$$

And then according to equation (11)

$$
\begin{aligned}
N_{1, X}= & E A * f_{1}\left(\frac{L}{H}, \frac{L}{D}, \ldots, \frac{d_{i}}{L}, \ldots\right) \\
& +\beta F t_{i}^{P} f_{2}\left(\frac{L}{H}, \frac{L}{D}, \ldots, \frac{d_{i}}{L}, \ldots\right)
\end{aligned}
$$

For the simplicity of the demonstration, one considers first that all cables have the same cross-sectional area $A_{c}$ and that all struts have the same cross-sectional area $A_{s}$. This assumption will be discussed and detailed in section "Generalization for struts with different cross-sectional areas." Equation (14) can be written as follows:

For a cable

$$
N_{1, X}=\left[\begin{array}{c}
\frac{E_{c} A_{c}}{F} * f_{1}\left(\frac{L}{H}, \frac{L}{D}, \ldots, \frac{d_{i}}{L}, \ldots\right) \\
+\beta t_{i}^{P} f_{2}\left(\frac{L}{H}, \frac{L}{D}, \ldots, \frac{d_{i}}{L}, \ldots\right)
\end{array}\right] F
$$

For a strut

$$
N_{1, X}=\left[\begin{array}{l}
\frac{E_{s} A_{s}}{F} * f_{1}\left(\frac{L}{H}, \frac{L}{D}, \ldots, \frac{d_{i}}{L}, \ldots\right) \\
+\beta t_{i}^{P} f_{2}\left(\frac{L}{H}, \frac{L}{D}, \ldots, \frac{d_{i}}{L}, \ldots\right)
\end{array}\right] F
$$

Node 1 being potentially subjected to an external load $F_{l, X}$ in the $X$ direction, its equilibrium in the $X$ direction can be written as $F_{1 X}=\sum N_{1 X}$, the summation being related to the extremity of all the elements having node 1 in common, using relations of type (15a) or (15b).

Doing the same reasoning for the $Y$ and $Z$ directions and for each node of the structure leads to the assembly of $3 * n$ equilibrium equations and the writing of the global $3 n \times 3 n$ matrix system under the generic form below. Note that the values of coefficients $t_{i}^{F}$ are supposed to be known, as far as the external load $\tilde{F}$ is supposed to be known

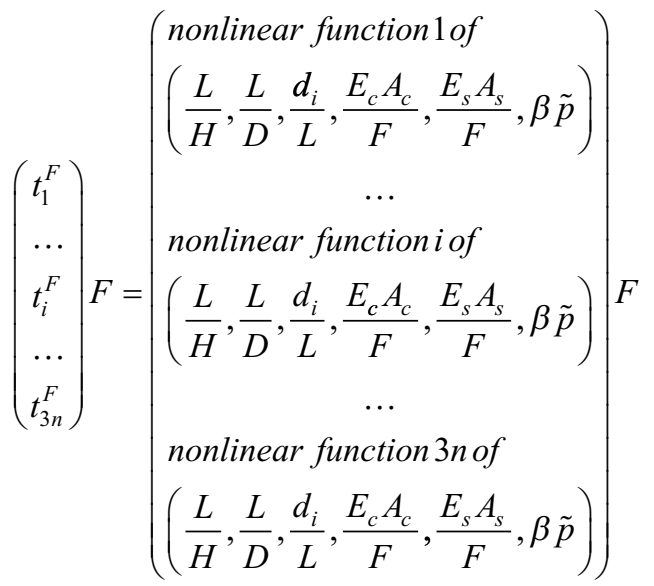

Inverting this system would lead to the following generic expression

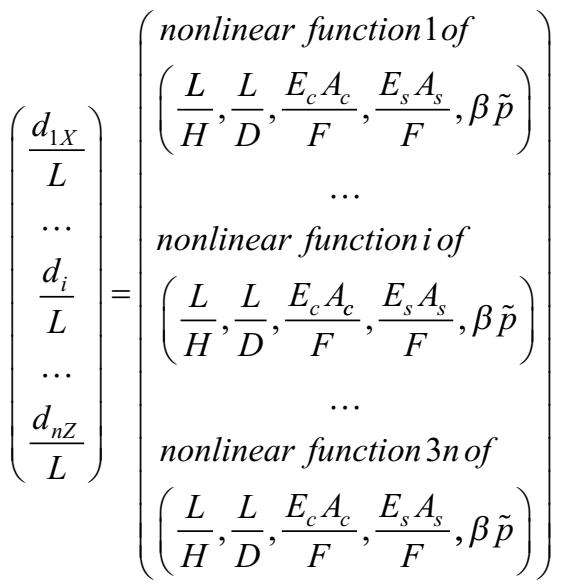

Solving previous system leads to the following generic form of each of the $3 n$ displacements $d_{i}$

$$
\frac{d_{i}}{L}=f\left(\frac{L}{H}, \frac{L}{D}, \frac{F}{E_{c} A_{c}}, \frac{F}{E_{s} A_{s}}, \beta \tilde{p}\right)
$$

The below developments aim at eliminating terms $F /\left(E_{c} A_{c}\right)$ and $F /\left(E_{s} A_{s}\right)$ from equation (17). 
Equation (17), introduced into equation (11), allows expressing the axial force in each of the $n_{c}+n_{b}$ element as follows

$$
\left\{\begin{array}{l}
\cdots \\
N_{c, i}=E_{c} A_{c} f\left(\frac{L}{H}, \frac{L}{D}, \frac{F}{E_{c} A_{c}}, \frac{F}{E_{s} A_{s}}, \beta \tilde{p}\right)+\beta F t_{i}^{P} \\
\cdots \\
N_{s, j}=E_{s} A_{s} \cdot\left(\frac{L}{H}, \frac{L}{D}, \frac{F}{E_{c} A_{c}}, \frac{F}{E_{s} A_{s}}, \beta \tilde{p}\right)+\beta F t_{j}^{P} \\
\cdots
\end{array}\right.
$$

Let us consider $N_{c, \max }$ as the highest value of the axial force in the cables. The design criterion is, according to equation (1a): $N_{c, \max }=\sigma_{c} A_{c}$

With equation (18), one obtains

$$
\frac{\sigma_{c}}{E_{c}}=f\left(\frac{L}{H}, \frac{L}{D}, \frac{F}{E_{c} A_{c}}, \frac{F}{E_{s} A_{s}}, \beta \tilde{p}\right)+\frac{F}{E_{c} A_{c}} \beta t_{i}^{P}
$$

And thus

$$
\frac{\sigma_{c}}{E_{c}}=f\left(\frac{L}{H}, \frac{L}{D}, \frac{F}{E_{c} A_{c}}, \frac{F}{E_{s} A_{s}}, \beta \tilde{p}\right)
$$

For the struts, the design criterion is given by equation (1b). If we assume that the form factor $q=I_{s} / A_{s}^{2}$ is the same for all the struts, and introducing it into equation (1b), one obtains

$$
\frac{N_{s, \max }}{A_{s}}=\sigma_{s}\left(1+\frac{\sigma_{s} L_{0}^{2}}{q \pi^{2} E_{s} A_{s}}\right)^{-1}
$$

And, the indicator of buckling having been defined previously as $\Psi=\sigma_{s} L / \sqrt{q E_{s} F}$, one obtains

$$
N_{s, \text { max }}=\frac{\sigma_{s} A_{s}}{1+\Psi^{2} \frac{1}{\pi^{2}}\left(\frac{L_{0}}{L}\right)^{2}\left(\frac{F}{\sigma_{s} A_{s}}\right)}
$$

Combining equations (18) and (20), one obtains

$$
\begin{aligned}
& f\left(\frac{L}{H}, \frac{L}{D}, \frac{F}{E_{c} A_{c}}, \frac{F}{E_{s} A_{s}}, \beta \tilde{p}\right)+\frac{F}{E_{s} A_{s}} \beta t_{j}^{P} \\
& =\frac{\sigma_{s} / E_{s}}{1+\Psi^{2} \frac{1}{\pi^{2}}\left(\frac{L_{0}}{L}\right)^{2}\left(\frac{F /\left(E_{s} A_{s}\right)}{\left(\sigma_{s} / E_{s}\right)}\right)}
\end{aligned}
$$

And thanks to equation (6), the previous equation can be written under the following generic form

$$
\frac{\sigma_{s}}{E_{s}}=f\left(\Psi, \frac{L}{H}, \frac{L}{D}, \frac{F}{E_{c} A_{c}}, \frac{F}{E_{s} A_{s}}, \beta \tilde{p}\right)
$$

Finally, both equations (19) and (21) lead to

$$
\left\{\begin{array}{l}
\frac{F}{E_{c} A_{c}}=f\left(\frac{L}{H}, \frac{L}{D}, \Psi, \frac{E_{c}}{\sigma_{c}}, \frac{E_{s}}{\sigma_{s}}, \beta \tilde{p}\right) \\
\frac{F}{E_{s} A_{s}}=f\left(\frac{L}{H}, \frac{L}{D}, \Psi, \frac{E_{c}}{\sigma_{c}}, \frac{E_{s}}{\sigma_{s}}, \beta \tilde{p}\right)
\end{array}\right.
$$

And, finally, combining equations (22) and (17) leads to

$$
\frac{d_{i}}{L}=f\left(\frac{L}{H}, \frac{L}{D}, \Psi, \frac{E_{c}}{\sigma_{c}}, \frac{E_{s}}{\sigma_{s}}, \beta \tilde{p}\right)
$$

In this equation, how to find the value of $\beta$ still needs to be discussed.

\section{Discussion about the value of $\beta$}

Concerning $\beta$, its minimum value $\beta_{\min }$ related to a situation where no cable slacks, that means where the least tensioned cable is related to $N=0$, can be found easily, thanks to equation (18). Indeed, considering in particular the smallest value $N_{c, \min }$ of $N_{c, i}$, equation (18) leads to

$$
\begin{aligned}
N_{c, \text { min }}= & E_{c} A_{c} \cdot f\left(\frac{L}{H}, \frac{L}{D}, \frac{F}{E_{c} A_{c}}, \frac{F}{E_{s} A_{s}}, \beta_{\min } \tilde{p}\right) \\
& +\beta_{\min } F t_{i}^{P}=0
\end{aligned}
$$

which leads to

$$
\beta_{\text {min }}=f\left(\frac{L}{H}, \frac{L}{D}, \frac{F}{E_{c} A_{c}}, \frac{F}{E_{s} A_{s}}, \tilde{p}\right)
$$

And thanks to equation (22)

$$
\beta_{\text {min }}=f\left(\frac{L}{H}, \frac{L}{D}, \Psi, \frac{E_{c}}{\sigma_{c}}, \frac{E_{s}}{\sigma_{s}}, \tilde{p}\right)
$$

Thanks to equation (24), and if $\beta=\beta_{\text {min }}$, equation (23) becomes

$$
\frac{d_{i}}{L}=f\left(\frac{L}{H}, \frac{L}{D}, \Psi, \frac{E_{c}}{\sigma_{c}}, \frac{E_{s}}{\sigma_{s}}, \tilde{p}\right)
$$

This ends the demonstration of equation (2a).

\section{Generalization for struts with different cross- sectional areas}

Equation (23) has been demonstrated assuming that all struts have the same cross-sectional area $A_{s}$, considering 
that the most stressed strut gives its cross section to all the others (and the same for the cables). Assuming that factor $q$ is the same for all struts (in order to consider a unique indicator of buckling $\Psi$ ), relation equation (23) is although still valid when the struts and the cables have each a different cross section designed, respectively, according to equations (1b) and (1a). Indeed, functions $f$ of equations (17) and (18) then contain $n_{c}$ terms $F /\left(E_{c} A_{c, i}\right)$ and $n_{s}$ terms $F /\left(E_{s} A_{s, i}\right)$.

Equation (21) can be written $n_{s}$ times for the struts, equation (19) can be written $n_{c}$ times for the cables, and one obtains $\left(n_{s}+n_{c}\right)$ equations allowing to find the $n_{c}$ terms $F /\left(E_{c} A_{c, i}\right)$ and the $n_{s}$ terms $F /\left(E_{s} A_{s, i}\right)$.

Note that the fully stressed design does not always find physically possible solutions and can lead to convergence problems of the numerical algorithm.

\section{Demonstration of relation $\sigma_{s} V / F L=f\left(L / H, L / D, \Psi, E_{c} / \sigma_{c}, E_{s} / \sigma_{s}, \tilde{p}, u\right)$ for a given $S$}

Thanks to the assumptions of section "Other assumptions," $0.99 \leq\left|L_{0} / L_{\text {ini }}\right| \leq 1.01$ the total volume of the structure is equal to

$$
V=\sum_{i=1}^{n_{c}} L_{0, i} A_{c, i}+\sum_{i=1}^{n_{s}} L_{0, i} A_{s, i}
$$

The previous relation can also be written as

$$
\frac{V}{F L}=\sum_{i=1}^{n_{c}}\left(\frac{L_{0, i}}{L}\right) \frac{A_{c, i}}{F}+\sum_{i=1}^{n_{s}}\left(\frac{L_{0, i}}{L}\right) \frac{A_{s, i}}{F}
$$

Or

$$
\begin{aligned}
\frac{V}{F L}= & \frac{1}{\sigma_{c}} \sum_{i=1}^{n_{c}}\left(\frac{L_{0, i}}{L}\right)\left(\frac{A_{c, i} E_{c}}{F}\right)\left(\frac{\sigma_{c}}{E_{c}}\right) \\
& +\frac{1}{\sigma_{s}} \sum_{i=1}^{n_{s}}\left(\frac{L_{0, i}}{L}\right)\left(\frac{A_{s, i} E_{s}}{F}\right)\left(\frac{\sigma_{s}}{E_{s}}\right)
\end{aligned}
$$

And, according to equations (6) and (22), one obtains

$$
\begin{aligned}
\frac{V}{F L}= & \frac{1}{\sigma_{c}} f\left(\frac{L}{H}, \frac{L}{D}, \Psi, \frac{E_{c}}{\sigma_{c}}, \frac{E_{s}}{\sigma_{s}}, \beta \tilde{p}\right) \\
& +\frac{1}{\sigma_{s}} f\left(\frac{L}{H}, \frac{L}{D}, \Psi, \frac{E_{c}}{\sigma_{c}}, \frac{E_{s}}{\sigma_{s}}, \beta \tilde{p}\right)
\end{aligned}
$$

And, finally, assuming that factor $u$ is defined as $u=\sigma_{c} / \sigma_{s}$, one obtains

$$
\frac{\sigma_{s} V}{F L}=f\left(\frac{L}{H}, \frac{L}{D}, \Psi, \frac{E_{c}}{\sigma_{c}}, \frac{E_{s}}{\sigma_{s}}, \beta \tilde{p}, u\right)
$$

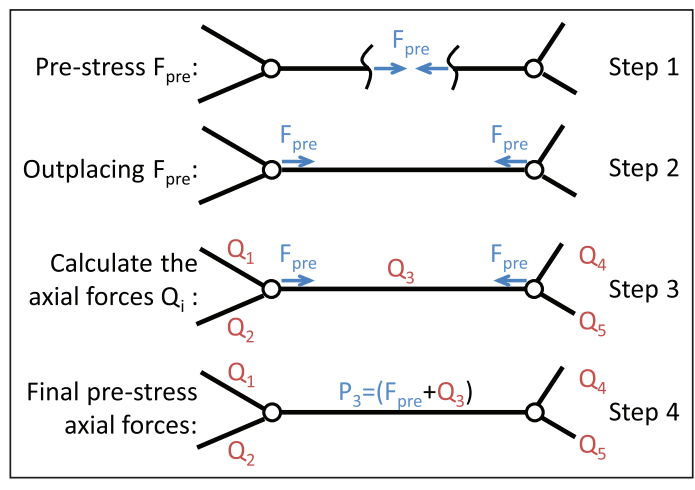

Figure 5. Considering a pre-stress as an external load.

And, if $\beta=\beta_{\text {min }}$, one finally obtains

$$
\frac{\sigma_{s} V}{F L}=f\left(\frac{L}{H}, \frac{L}{D}, \Psi, \frac{E_{c}}{\sigma_{c}}, \frac{E_{s}}{\sigma_{s}}, \tilde{p}, u\right)
$$

This ends the demonstration of equation (2b).

\section{Choice of a pre-stress scenario $\tilde{\boldsymbol{P}}$}

Equations (25) and (26) show that the displacements and the volume of a structure depend on the pre-stress state $\tilde{p}$ , although the search for the best pre-stress state $\tilde{p}$ is not the aim of this article. Furthermore, considering that $\tilde{p}$ is a self-stress state is not necessary a good choice for several reasons: the optimization algorithm would become extremely complex, $\tilde{p}$ depends itself of many parameters (among which, $L / H$ ), and mainly, a self-stress state is not necessary the one that leads to a good structural behavior with respect to the particular external load $\tilde{F}$. It seems thus relevant to search for a way of finding $\tilde{p}$ that guaranties that it is only a function of the same parameters $\left(L / H, L / D, \Psi, E_{c} / \sigma_{c}, E_{s} / \sigma_{s}\right)$.

For instance, a way to numerically simulate a pre-stress into a structure is to apply an external axial force $F_{\text {pre }}$ at both extremities of an element, as shown in Figure 5. Introducing $F_{\text {pre }}$ into this element will lead, after calculation, to a situation where each element of the structure, including the one in which $F_{\text {pre }}$ was initially introduced as external load, is finally subjected to a force $Q_{i}$ different from $F_{\text {pre }}$.

The previous reasoning can be extended: a way to numerically create a pre-stress scenario $\tilde{P}$ is to numerically apply to each element of the structure an external axial force $F_{p r e, i}$ at its extremities.

The $\left(n_{c}+n_{s}\right)$ values of the initial axial force $F_{p r e, i}$ can be defined by vector $\tilde{F}_{\text {pre }}$

$\tilde{F}_{\text {pre }}=\tilde{f}_{\text {pre }}\left(\beta_{\text {pre }} F\right)=\left(t_{1}^{\text {pre }}, t_{2}^{\text {pre }}, \ldots, t_{i}^{\text {pre }}, \ldots, t_{n_{c}+n_{s}}^{\text {pre }}\right)\left(\beta_{\text {pre }} F\right)$,

with $\left\{\begin{array}{l}-1 \leq \tilde{f}_{\text {pre }}=\left(t_{1}^{\text {pre }}, t_{2}^{\text {pre }}, \ldots, t_{i}^{\text {pre }}, \ldots, t_{n_{c}+n_{s}}^{\text {pre }}\right) \leq 1 \\ \beta_{\text {pre }} \geq 0\end{array}\right.$ 


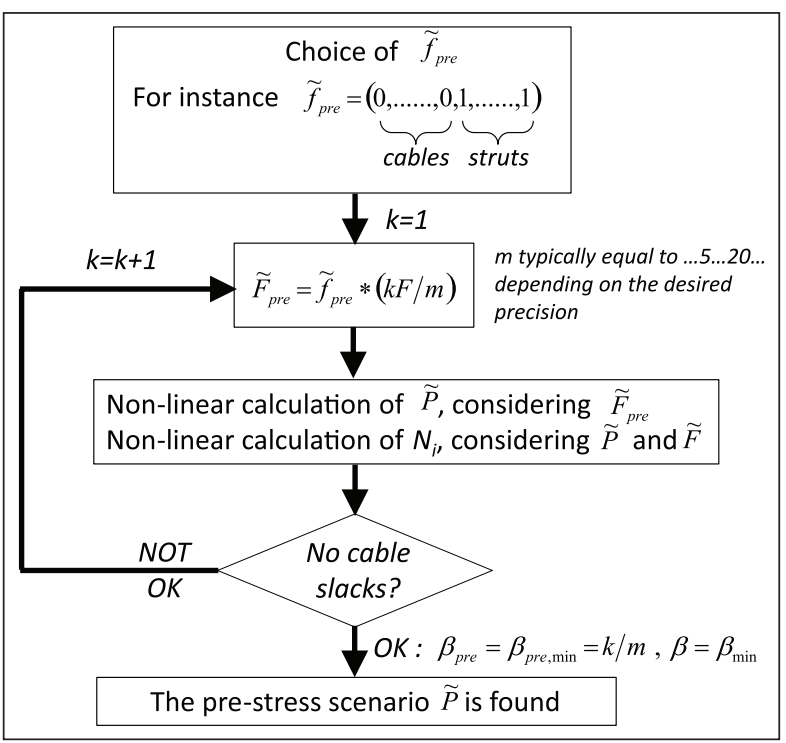

Figure 6. Finding the pre-stress scenario $\tilde{P}$.

$\beta_{\text {premin }}$, just like $\beta_{\text {min }}$, is defined as being the particular value of $\beta_{\text {pre }}$ that leads to a situation where the axial force in the least tensioned cable after application of the external load $\tilde{F}$ is equal to zero, which means that no cable slacks. As shown in section "Discussion about the value of $\beta$ " by relation (24), they both only depend on parameters $(L / H$, $\left.L / D, \Psi, E_{c} / \sigma_{c}, E_{s} / \sigma_{s}, \tilde{p}\right)$.

The proposed design methodology considers that the choice of $\tilde{f}_{\text {pre }}$ is arbitrary, chosen once for all. But the designer must be aware that, on one hand, the chosen vector $\tilde{f}_{\text {pre }}$ does not necessarily lead to a solution where no cable slacks, and, on the other hand, there may exist a better choice, which leads to a stiffer or lighter structure. For the examples discussed in sections "Numerical confirmation of equations (28) and (29)," "Example of curves of efficiency," and "Other examples: trusses and other tensegrity topologies," one considers that the values of $\tilde{f}_{\text {pre }}$ are null for the cables and identical for the struts $f_{\text {pre }}=(0, \ldots, 0,1, \ldots, 1)$. This hypothesis corresponds to a practical situation where only struts are equipped with a mechanical device (that can elongate them).

Figure 6 shows a way to find $\tilde{F}_{\text {pre }}$ and its associate prestress scenario $\tilde{P}$, which is compatible with the external load case $\tilde{F}$.

The final step of the demonstration is to prove that if $\tilde{f}_{\text {pre }}$ is chosen and fixed once for all, the pre-stress state $\tilde{p}$ only depends on parameters $\left(L / H, L / D, \Psi, E_{c} / \sigma_{c}, E_{s} / \sigma_{s}\right)$, which is useful to get rid of $\tilde{p}$ in relations (2a), (2b), and (24)-(26) and finally demonstrate the validity of equations (3a) and (3b).

For this purpose, let us apply the developments of section "Demonstration of relation $\delta / L=f\left(L / H, L / D, \Psi, E_{c} / \sigma_{c}, E_{s} / \sigma_{s}, \tilde{p}\right)$ for a given
$S$," this time not considering the phase where $\tilde{F}$ is applied after an existing $\tilde{P}$, but the phase where $\tilde{F}_{\text {pre }}$ is applied alone and creates $\tilde{P}$. In this case, relation (18) can be rewritten as follows, where step 4 of Figure 5 is responsible for adding the last term $t_{i}^{\text {pre }} \beta_{\text {pre }} F$

$$
\begin{aligned}
& P_{i}=t_{i}^{P} \beta F=E_{\text {cor } ~} A_{\text {cors }} f \\
& \left(\frac{L}{H}, \frac{L}{D}, \frac{\beta_{\text {pre }} F}{E_{c} A_{c}}, \frac{\beta_{\text {pre }} F}{E_{s} A_{s}}, 0\right)+0+t_{i}^{\text {pre }} \beta_{\text {pre }} F
\end{aligned}
$$

This leads to

$$
t_{i}^{P} \beta=f\left(\beta_{\text {pre }}, \frac{L}{H}, \frac{L}{D}, \frac{F}{E_{c} A_{c}}, \frac{F}{E_{s} A_{s}}\right)
$$

This equation represents thus the $\left(n_{c}+n_{s}\right)$ axial forces of vector $\tilde{P}$ into the structure after the application of $\tilde{F}_{\text {pre }}$. Thanks to equations (22) and (24), previous equation gives

$$
\begin{aligned}
t_{i}^{P} & =f\left(\frac{L}{H}, \frac{L}{D}, \Psi, \frac{E_{c}}{\sigma_{c}}, \frac{E_{s}}{\sigma_{s}}\right) \text { and } \\
\tilde{p} & =f\left(\frac{L}{H}, \frac{L}{D}, \Psi, \frac{E_{c}}{\sigma_{c}}, \frac{E_{s}}{\sigma_{s}}\right)
\end{aligned}
$$

Thanks to equation (27), relations (25) and (26) can thus get rid of vector $\tilde{p}$ and be rewritten as follows

$$
\begin{gathered}
\frac{d_{i}}{L}=f\left(\frac{L}{H}, \frac{L}{D}, \Psi, \frac{E_{c}}{\sigma_{c}}, \frac{E_{s}}{\sigma_{s}}\right) \\
\frac{\sigma_{s} V}{F L}=f\left(\frac{L}{H}, \frac{L}{D}, \Psi, \frac{E_{c}}{\sigma_{c}}, \frac{E_{s}}{\sigma_{s}}, u\right)
\end{gathered}
$$

This ends the demonstration of equations (3a) and (3b).

\section{Optimization algorithm}

Figure 7 summarizes the algorithm that the authors used to bring a numerical confirmation of the validity of equations (28) and (29), illustrated by examples of section "Numerical confirmation of equations (28) and (29)." Once the material is chosen $\left(E_{c}, \sigma_{c}, E_{s}, \sigma_{s}\right)$ and $\tilde{f}_{\text {pre }}$ chosen, the algorithm allows finding, for a given $S$, the value of $L / H$ that corresponds to the minimum deflection $\delta / L$ at mid-span (but any other deflection could be considered) for a given value of the indicator of buckling $\Psi$. The best (the minimum) value of $\delta / L$ is numerically easy to find as it is just the result of a search for the minimum value among the solutions given for each $L / H$. 


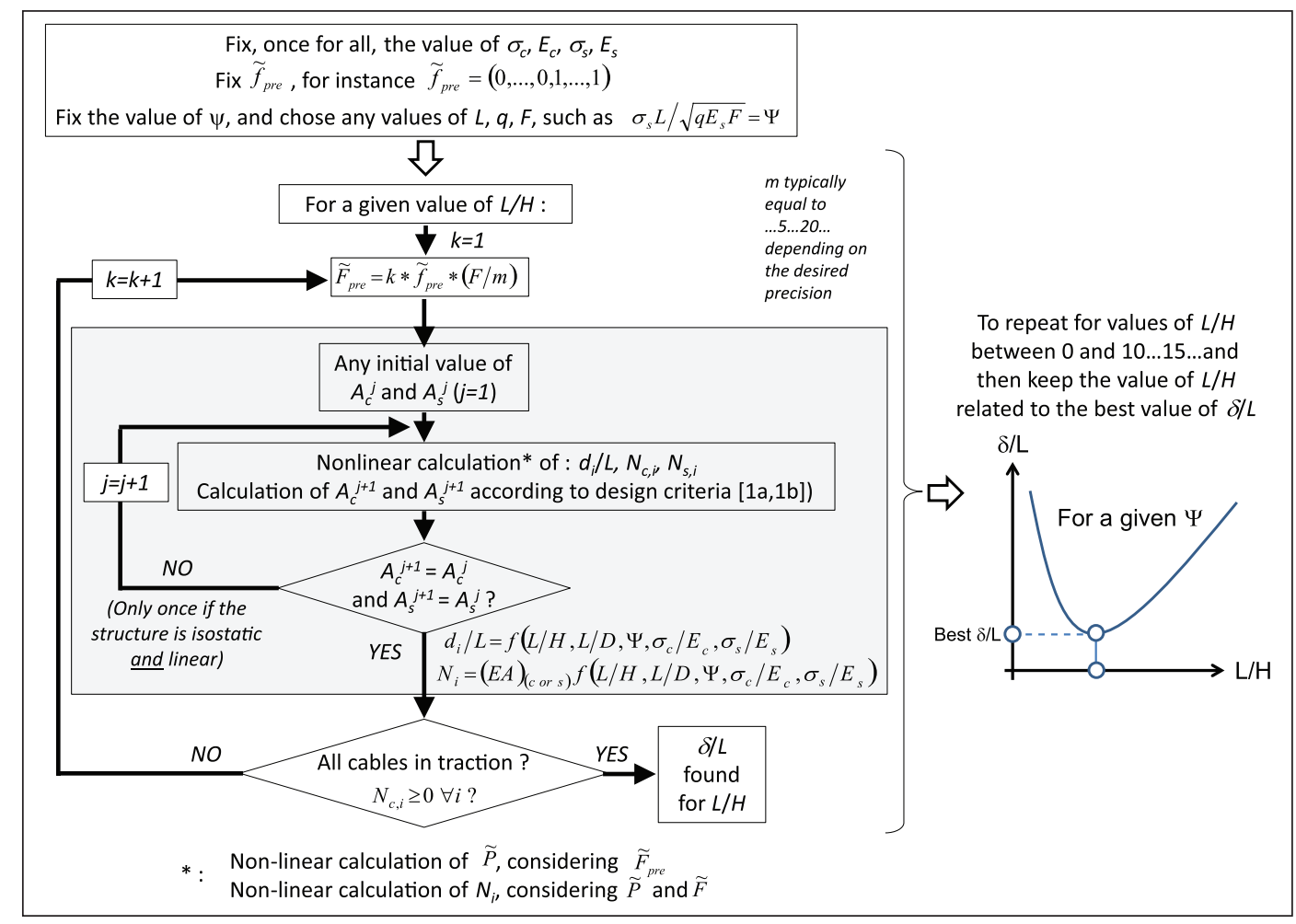

Figure 7. General algorithm to find the value of $\left(L / H,(\delta / L)_{\text {min }}\right)$ for a given value of $\Psi$ (here, $A_{s}$ is the same cross-sectional area for all struts and $A_{c}$ is the same cross-sectional area for all cables).

Algorithm of Figure 7 can be used for several values of $S$, for instance, $2,3,4,5,6$, and so on. For a given value of $S$, the algorithm has to be repeated for values of $\Psi$ between 0 and 100 . Indeed, previous studies ${ }^{17-19}$ have shown that values of $\Psi$ bigger than 100 are related to very heavy and low-efficient structures. Note that the algorithm is similar if the objective is to minimize the volume (via relation (29)).

\section{Numerical confirmation of equations (28) and (29)}

In this section, we intend to numerically confirm the validity of relations (28) and (29) using the algorithm of Figure 7. For this purpose, we have chosen to generate the pre-stress scenario, thanks to identical axial forces introduced as an external force at each extremity of the struts

$$
\begin{aligned}
& \tilde{F}_{\text {pre }} \stackrel{\text { proportionnalto }}{\approx}(0, \ldots, \ldots, 0,1, \ldots, 1) \\
& * F, \quad(0 \text { :for cables }, 1 \text { :for struts })
\end{aligned}
$$

Figure 8 shows a tensegrity beam of span $10 \mathrm{~m}$ subjected to an external load of $30 \mathrm{kN}$, composed of four simplex modulus, with an hyperstaticity equal to 1 . Figure 9 shows another tensegrity beam composed of four simplex modulus with a hyperstaticity equal to 1 , but this time of span $30 \mathrm{~m}$ and subjected to an external load of $125 \mathrm{kN}$. Supports are shown with black arrows on the lower extremity nodes. It is important to precise that both structures have (arbitrary choice) an indicator of buckling equal to $\Psi=50$ and a value $E / \sigma=894$ for both cables and struts.

Graphs of Figure 10 are related to the structure of Figure 8 for a particular value of $H=2 \mathrm{~m}(L / H=5)$. The left graph shows the various axial forces $P_{i}$ calculated in the cables and the struts under initial axial forces $F_{\text {pre }}=140 \mathrm{kN}$ numerically introduced into the struts and with no external load. In other words, one has $\tilde{F}_{\text {pre }}=(0, \ldots, 0,1, \ldots, 1) *\left(\beta_{\text {pre }}=140 / 30\right) * F$. The value of $140 \mathrm{kN}$ was reached for $\beta_{\text {pre }}=\beta_{\text {pre, } \min }$ and $\beta=\beta_{\text {min }}$ (situation where the least tensioned cable is related to $N=0$ ).

The right graph shows internal forces due to $\vec{F}_{p r e}$ combined with the external load $F=30 \mathrm{kN}$. The algorithm of Figure 7 fitted the value of $\vec{F}_{\text {pre }}$ and $\beta_{\text {pre }}$ in such way that no cable slacks. It is cable 21 that reaches the minimum value of $N$ when $\tilde{F}$ is applied, followed by cables 33 and 36 .

Figure 11, resulting from a numerical calculation according to the organigram of Figure 7 and, this time, for a large range of values $L / H$, shows that both structure 1 and structure 2 having the same $S$, the same pre-stressing initial scenario $\tilde{f}_{\text {pre }}=(0, \ldots, 0,1, \ldots, 1)$, the same values of $\Psi=50$ and $E / \sigma=894$ show exactly the same curves 
$L / \delta-L / H$ (left curve) and the same curves $W-L / H$ (right curve). This brings the numerical confirmation of equations (27)-(29).

These kinds of curves can also lead to interesting considerations, such as in this particular case:

- The best stiffness is related to $L / H=12$ and varies little for $L / H>8$;

- For this particular choice of $\tilde{f}_{\text {pre }}$, it is impossible to obtain a value of $L / \delta$ better than 267, unless the prestress is increased beyond the strict necessary value $\beta=\beta_{\text {min }}(\theta>1)$, or the value of $E_{s} / \sigma_{s}$ or $E_{c} / \sigma_{c}$ is increased, or the value of $\Psi$ is increased as shown in Figure 12, that means reducing the value of factor $q$

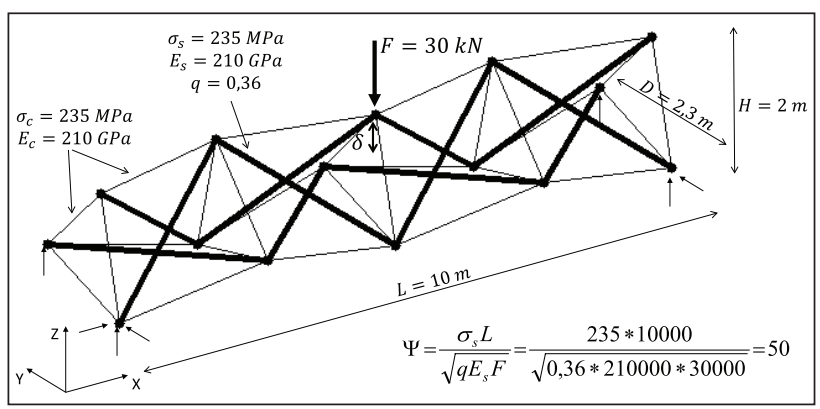

Figure 8. Structure I.

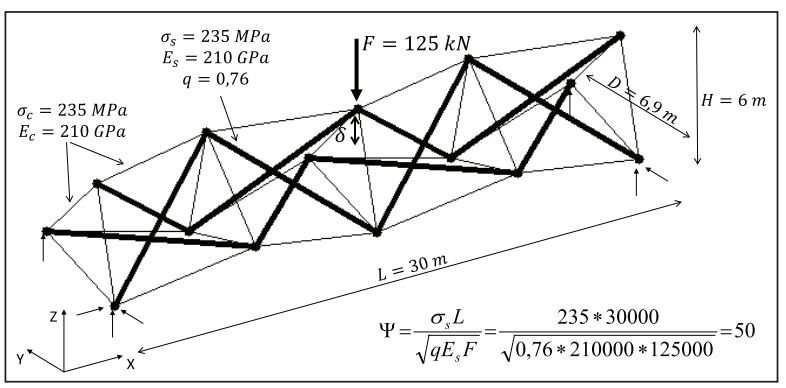

Figure 9. Structure 2. of the cross section or eventually increasing the number $S$ of Simplex modulus (see section "Example of curves of efficiency").

- The minimum volume $V$ is related to $L / H=7$ and is equal to:

- For the 10-m span structure: $V=(F L / \sigma)^{*} W=3$ $0,000 * 10,000 / 235 * 48.3=61.6 \times 10^{6} \mathrm{~mm}^{3}$, that means a self-weight equal to $4,8 \mathrm{kN}$;

- For the 30-m span structure: $V=(F L / \sigma)^{*} W=12$ $5,000 * 30,000 / 235 * 48.3=770.7 \times 10^{6} \mathrm{~mm}^{3}$, that means a self-weight equal to $60.5 \mathrm{kN}$.

\section{Example of curves of efficiency}

The structures of Figures 8 and 9 have been computed for values of the indicator of buckling $\Psi$ between 0 and 70 in order to find, in each case, the minimum value of $\delta / L$ (or maximum $L / \delta$ ). The result is given in Figure 12, which shows the curve of efficiency of $L / \delta$ when the structure is composed of $S=4,6$, and 8 elementary tensegrity modulus. The efficiency curves of $W$ could also be drawn the same way, which could also bring useful information to the designer.

In this particular example, the curve of efficiency of Figure 12 shows that

- Whatever the value of $\Psi$, the best values of $L / H$ are, respectively, for $S=4, S=6$, and $S=8$, related to values between 11 and 12,12 and 13 , and 13 and 14 . In other words, the higher the $S$, the larger the $L / H$.

- The higher the $\Psi$ (that means reducing the value of factor $q$ ), the more the stiffness increases (better ratio $L / \delta$ ).

- Increasing the number $S$ leads to a better stiffness.

- Coming back to the structures of Figures 8 and 9 related to $S=4, \Psi=50$, and $E / \sigma=894$, the best stiffness corresponds to $L / \delta=267$ and $L / H=12$, as also shown in Figure 11 (left).

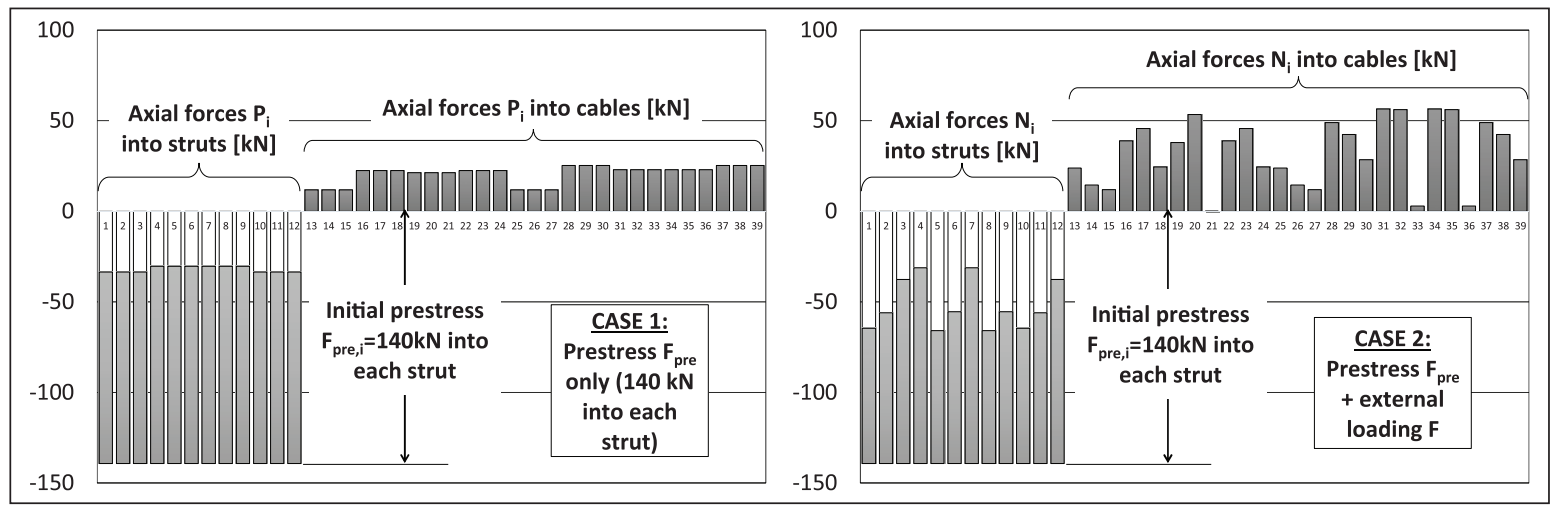

Figure I0. Values of the axial forces for structure I (Figure 8, $S=4, \Psi=50, E / \sigma=894, L / H=5$ ). 


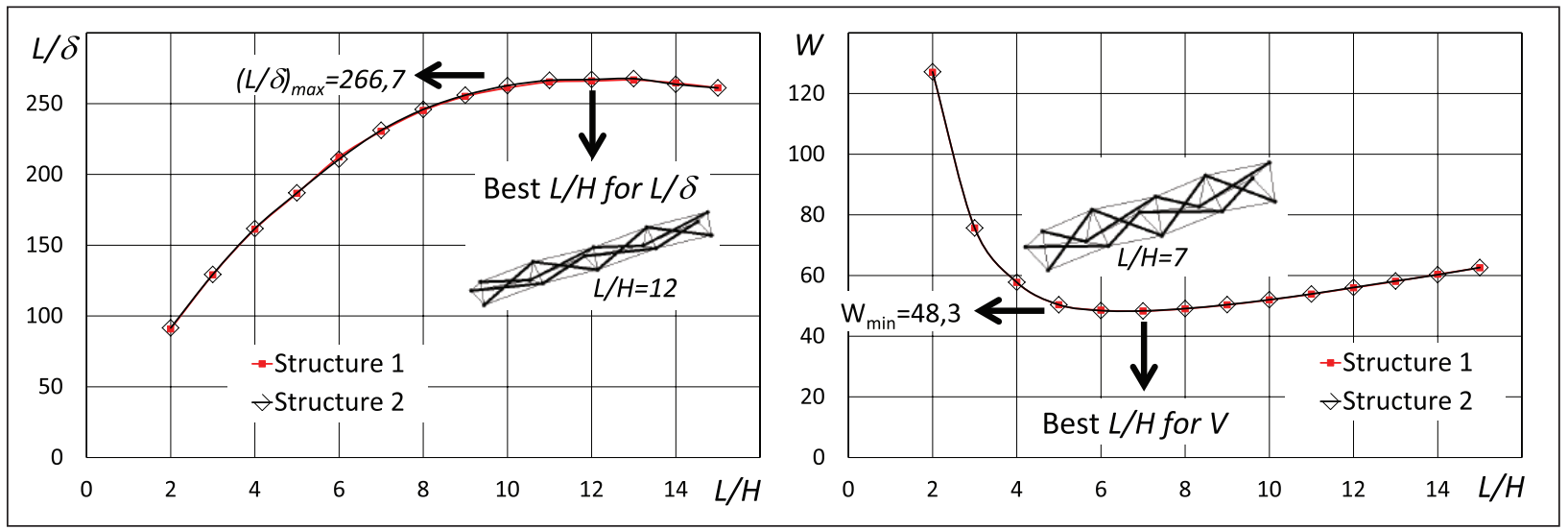

Figure II. The values of $L / \delta$ and $W$ are identical for both structures related to the same $S, \Psi, E_{s} / \sigma_{s}, E_{c} / \sigma_{c}, u$, and $\tilde{f}_{\text {pre }}$.

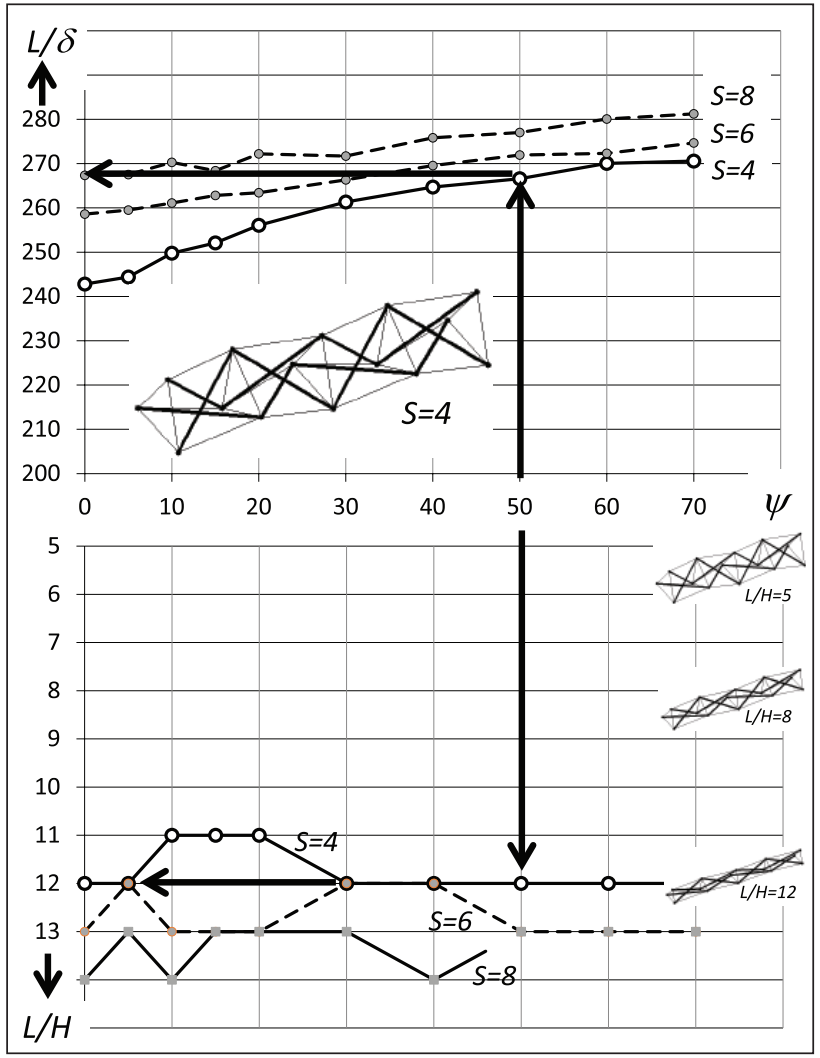

Figure 12. Curves of efficiency of $L / \delta(E / \sigma=894$ for all cables and struts).

\section{Other examples: trusses and other tensegrity topologies}

In this section are shown the results given by the design methodology for two other topologies of structures. The first structure is a "usual" truss (linear behavior, no prestress) composed of four pyramidal modulus (Figure 13). The second one is tensegrity based, but this time composed of quadruplex modulus (Figure 14). To allow the

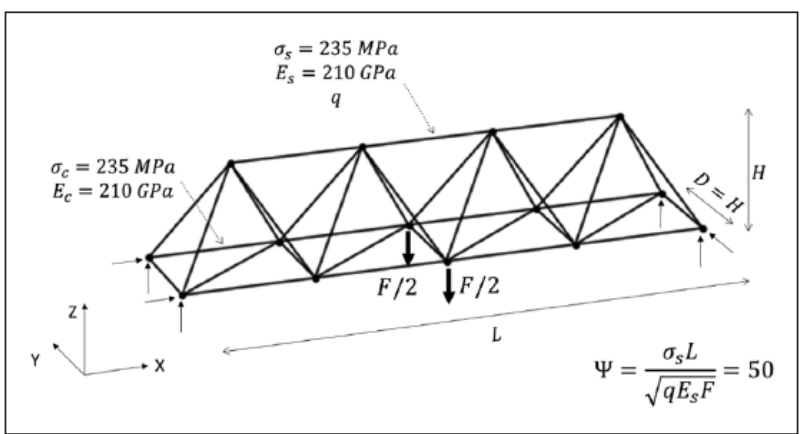

Figure 13. Truss (linear behavior, no pre-stress).

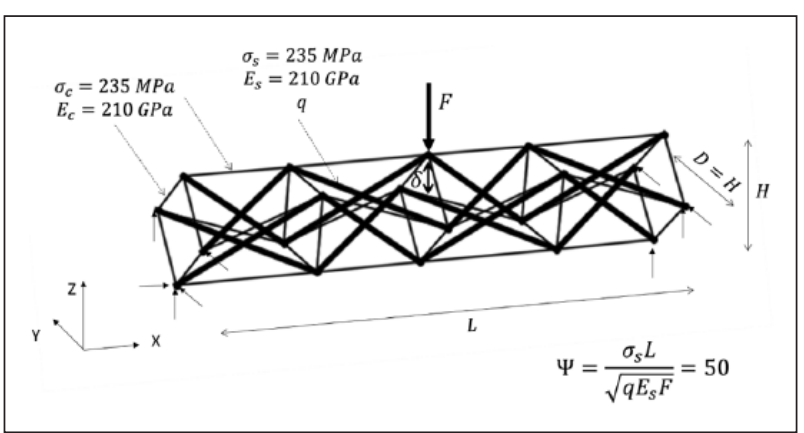

Figure 14. Tensegrity based composed of quadruplex modulus.

comparison with the topology previously studied (Figure 8 or 9), the same kind of external load case is considered (centered $F$ ). Moreover, the three structures (Figures 8 or 9 , 13, and 14) are related to the same values of $S, \Psi$, $E_{s} / \sigma_{s}, E_{c} / \sigma_{c}, u$, and $\tilde{f}_{\text {pre }}$, if pre-stressed.

Figure 15 is given for arbitrary values of $E / \sigma(=894)$ and the indicator of buckling $(=50)$ and illustrates the power of the design methodology. Indeed, it shows that for these three families and with the particular type of load case and the values of $S, \Psi, E_{s} / \sigma_{s}, E_{c} / \sigma_{c}, u, \tilde{f}_{\text {pre }}$ : 


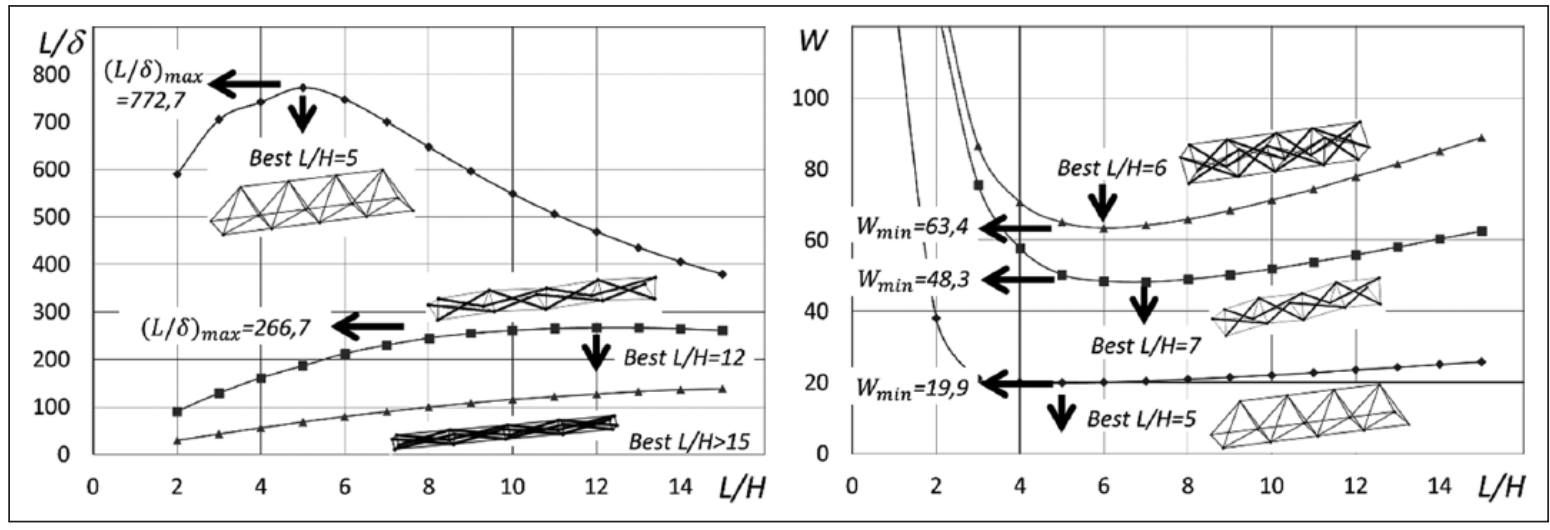

Figure 15. Comparison of $L / \delta$ and $W$ for the three topologies (truss, simplex, and quadruplex) related to the same $S$, $\Psi$, $E_{s} / \sigma_{s}, E_{c} / \sigma_{c}, u, \tilde{f}_{\text {pre }}$.

- The "Simplex topology" is always stiffer and lighter than the "Quadruplex topology."

- The best truss is more than 2 times stiffer and 2 times lighter than the best tensegrity topology.

\section{Conclusion and discussion}

The study has extended the validity of the theory of morphological indicators to 3D hyperstatic pre-stressed and nonlinear structures. It has been proved that any displacement $d_{i}$, in particular the deflection $\delta$ at mid-span, and the volume $V$ of materials of any structure designed according to equations (1a) and (1b) are such that[AQ: 3]

$$
\begin{gathered}
\frac{\delta}{L}=f\left(\frac{L}{H}, \frac{L}{D}, \Psi, \frac{E_{c}}{\sigma_{c}}, \frac{E_{s}}{\sigma_{s}}, S, \tilde{p}\right) \\
\frac{\sigma_{s} V}{F L}=f\left(\frac{L}{H}, \frac{L}{D}, \Psi, \frac{E_{c}}{\sigma_{c}}, \frac{E_{s}}{\sigma_{s}}, S, \tilde{p}, u\right)
\end{gathered}
$$

For a given $S$, these relations become, as soon as the materials and the initial pre-stressing scenario $\tilde{f}_{\text {pre }}$ are chosen

$$
\begin{gathered}
\frac{\delta}{L}=f\left(\frac{L}{H}, \frac{L}{D}, \Psi\right) \\
\frac{\sigma_{s} V}{F L}=f\left(\frac{L}{H}, \frac{L}{D}, \Psi\right)
\end{gathered}
$$

Equations (30) and (31) can greatly simplify the numerical algorithms used for the search for the stiffest or the lightest structures. They can be used for any family of structures in order to draw the efficiency curves and to find the best solutions or simply to compare the efficiency of structures belonging to different families. As explained in sections "Pre-stress" and "Choice of a pre-stress scenario $\tilde{P}$," this design methodology requires the designer to first arbitrarily choose one initial pre-stress scenario $\tilde{f}_{\text {pre }}$ which may not be the best one. Nevertheless, it allows comparing different solutions coming from different choices of $\tilde{f}_{\text {pre }}$. The comparison of the solutions given in terms of displacements and volume for several choices of $\tilde{f}_{\text {pre }}$, leading or not to a self-stress state, would be an interesting following of this research.

It has also been proved that hyperstaticity does not change the generic expressions (30) and (31), while the nonlinear behavior is responsible for the presence of terms $E_{c} / \sigma_{c}$ and $E_{s} / \sigma_{s}$. If the structure has a linear behavior and in case of a single material for cables and struts, the relations get simplified and become the expressions of the indicator of displacement and the indicator of volume developed in Latteur ${ }^{18}$ for trusses, cables, and arches

$$
\frac{E \delta}{\sigma L}=f\left(\frac{L}{H}, \frac{L}{D}, \Psi\right) \text { and } \frac{\sigma V}{F L}=f\left(\frac{L}{H}, \frac{L}{D}, \Psi\right)
$$

In case of 2D linear structures not subjected to buckling, the upper relations get again simplified and lead to the expressions similar to the ones used by Zalewski and Kus, ${ }^{13}$ Quintas Ripoll, ${ }^{14,15}$ and Samyn ${ }^{16,17}$

$$
\frac{E \delta}{\sigma L}=f\left(\frac{L}{H}\right) \text { and } \frac{\sigma V}{F L}=f\left(\frac{L}{H}\right)
$$

The examples of section "Numerical confirmation of equations (28) and (29)" have shown that for large spans, the self-weight can become important compared to the external loads. Self-weight then acts as a new load case of total value $\rho V$, which should be combined with the external load case. In this case, the 


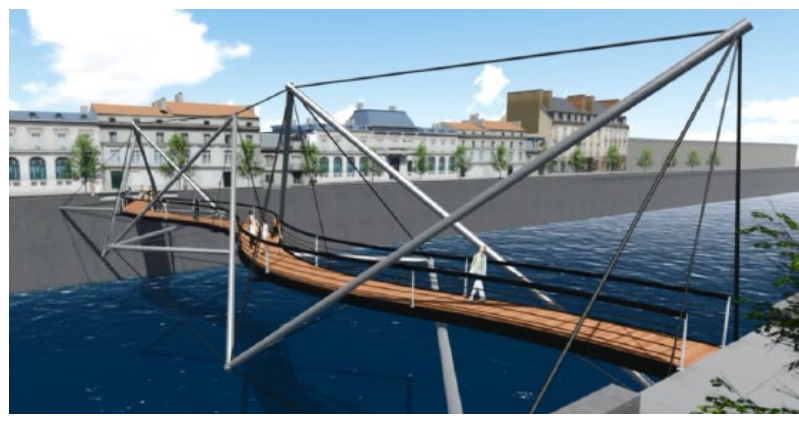

Figure 16. An example of tensegrity footbridge.

optimization process has to take into account a new parameter equal to $\rho L / \sigma_{s}$, called indicator of selfweight, ${ }^{18}$ and the algorithm of Figure 7 has to be adapted with a new iterative process.

This theory seems promising as it opens the door for fast and rigorous designs based on stiffness and volume optimization, not only of tensegrity structures but also of any kinds of 3D lattices and structures composed of prestressed elements. It also allows a fast stiffness and volume comparison of tensegrity structures with other kinds of classical structures. Further research could, however, include the consideration of random load cases and dynamic loads and thus extend considerably the relevance of the design methodology. Using this theory, the authors now intend investigating different tensegrity topologies that would allow building footbridges with a sufficient stiffness and a minimum volume. Figure 16 shows a footbridge that could be further studied and optimized using this design tool.

\section{Acknowledgements}

The authors wish to thank René Motro for the exchanges and the useful advices he gave when they decided to investigate the use of morphological indicators for tensegrity structures.

\section{Declaration of conflicting interests}

The author(s) declared no potential conflicts of interest with respect to the research, authorship, and/or publication of this article.

\section{Funding}

The author(s) received no financial support for the research, authorship, and/or publication of this article.

\section{References}

1. Skelton RE and De Oliveira M. Tensegrity systems. Berlin: Springer Science+Business Media, 2009.

2. Tibert AG and Pellegrino S. Review of form-finding methods for tensegrity structures. Int J Solids Struct 2003; 38: 5223-5252.

3. Tran HC and Lee J. Advanced form-finding of tensegrity structures. Comput Struct 2010; 88: 237-246.
4. Zhang JY and Ohsaki M. Adaptative force density method for form-finding problem of tensegrity structures. Int $J$ Solids Struct 2006; 43: 5658-5673.

5. Gomez Estrada G, Bungartz H-J and Mohrdieck C. Numerical form-finding of tensegrity structures. Int J Solids Struct 2006; 43: 6855-6868.

6. De Jager B and Skelton E. Symbolic stiffness optimization of planar tensegrity structures. J Intel Mat Syst Str 2004; 15: 181-193.

7. Masic M, Skelton RE and Gill PE. Optimization of tensegrity structures. Int J Solids Struct 2006; 43: 4687-4703.

8. Rhode-Barbarigos L, Bel Hadj Ali N, Motro R, et al. Designing tensegrity modules for pedestrian bridges. Eng Struct 2010; 32: 1158-1167.

9. Bel Hadj Ali N, Rhode-Barbarigos L, Pascual Albi A, et al. Design optimization and dynamic analysis of a tensegritybased footbridge. Eng Struct 2010; 32: 3650-3659.

10. Zalewski W. The flow of forces. In: Excerpt from notes on structural behavior for architecture students. MIT Press, 1980.[AQ: 4]

11. Michell AGM. The limit of economy of material in frame structures. Philos Mag 1904; 8(47): 589-597.

12. Skelton RE and De Oliveira MC. Optimal tensegrity structures in bending: the discrete Michell Truss. J Frankl Inst 2010; 347: 257-283.

13. Zalewski W and Kus S. Shaping structures for least weight. In: Actes du congrès international de l'IASS à Stuttgart, 1996, pp. 376-383.[AQ: 5]

14. Quintas Ripoll V. Sobre el teorema de Maxwell y la optimization de arcos de cubierta. Inf Constr 1989; 40(400): $57-70$.

15. Quintas Ripoll V. Sobre las formas de minimo volumen de las celosias de seccion constante. Inf Constr 1992; 43(418): 61-67.

16. Samyn P. Etude comparée du volume et du déplacement de structures isostatiques bidimensionnelles sous charges verticales entre deux appuis. Thèse de doctorat, Université de Liège, Liège, 2000.

17. Samyn P. Etude de la morphologie des structures à l'aide des indicateurs de volume et de déplacement. Publication de la Classe des Sciences de l'Académie Royale de Belgique, collection in $4^{\circ}, 3$ e série, tome $\mathrm{V}, 2004$.

18. Latteur P. Optimisation et prédimensionnement des treillis, arcs, poutres et câbles sur base d'indicateurs morphologiques. Application aux structures soumises en partie ou en totalité au flambement. Thèse de doctorat, Vrije Universiteit Brussel, Brussel, 2000.

19. Van Steirteghem J. A contribution to the optimisation of structures using morphological indicators: (in)stability and dynamics. Thèse de doctorat, Vrije Universiteit Brussel, Brussel, 2006.

20. Vandenbegh T. Benchmarking optimization at conceptual design stage with morphological indicators. $\mathrm{PhD}$ Thesis, Vrije Universiteit Brussel, 2010.

21. Vandenbergh $\mathrm{T}$ and De Wilde P. A review on conceptual design with morphological indicators. Int J Struct Eng 2010; 1(3/4): 280-298.

22. Zhang $P$ and Feng J. Initial prestress design and optimization of tensegrity systems based on symmetry and stiffness. Int J Solids Struct 2017; 106-107: 68-90. 
23. Ashwear N, Tamadapu $\mathrm{G}$ and Eriksson A. Optimization of modular tensegrity structures for high stiffness and frequency separation requirements. Int J Solids Struct 2016; 80: 297-309.

\section{Appendix I}

\section{Notation}

$a, b, c$ dimensionless numbers, respectively, equal to $\left(X_{2}-X_{1}\right) / L,\left(Y_{2}-Y_{1}\right) / D$, and $\left(Z_{2}-Z_{1}\right) / H$

A cross-sectional area

$A_{c}$ cross-sectional area of a cable

$A_{c, i} \quad$ cross-sectional area of a cable of index $i$

$A_{c}^{j} \quad$ cross-sectional area of the cables (all the same) at step $j$ of the iteration process

$A_{c}^{j+1} \quad$ cross-sectional area of the cables (all the same) at step $j+1$ of the iteration process

$A_{s}$ cross-sectional area of a strut

$A_{s, i} \quad$ cross-sectional area of a strut of index $i$

$A_{s}^{j} \quad$ cross-sectional area of the struts (all the same) at step $j$ of the iteration process

$A_{s}^{j+1} \quad$ cross-sectional area of the struts (all the same) at step $j+1$ of the iteration process

$c_{d e f, X}, c_{d e f, Y}, c_{d e f, Z}$ equal to, respectively, $\cos \alpha_{d e f, X}, \cos$

$\alpha_{d e f, Y}, \cos \alpha_{d e f, Z}$

$c_{X}, c_{Y}, c_{Z}$ equal to, respectively, $\cos \alpha_{X}, \cos \alpha_{Y}, \cos \alpha_{Z}$

$d_{i} \quad$ can be $d_{i X}$ or $d_{i Y}$ or $d_{i Z}$

$\left(d_{i X}, d_{i Y}, d_{i Z}\right)$ the displacements at node $i$, expressed in the $(X, Y, Z)$ system of axis

$D$ width of the structure before application of external load $\tilde{F}$

E Young's modulus

$E_{c} \quad$ Young's modulus of the cables' material

$E_{s} \quad$ Young's modulus of the struts' material

$f, f_{1}, f_{2}$ undefined functions

$\tilde{f} \quad$ vector $\left(t_{1}^{F}, t_{2}^{F}, t_{3}^{F}, \ldots, t_{3 n}^{F}\right)$, with $-1 \leq t_{i}^{F} \leq 1$ and $\sum_{\tilde{f}_{\text {pre }}}\left|t_{i}^{F}\right|=1$

$-1 \leq t_{i}^{\text {pre }} \leq 1$

$F \quad$ see definition of $\tilde{F}$

$\tilde{F}=\left(F_{1, X}, F_{1, Y}, F_{1, Z}, \ldots, F_{Z, 3 n}\right)=\left(t_{1}^{F}, t_{2}^{F}, t_{3}^{F}, \ldots, t_{3 n}^{F}\right) * F$, with $-1 \leq t_{i}^{F} \leq 1$ and $\sum\left|t_{i}^{F}\right|=1$, is the external load acting on each node
$F_{\text {pre }}, F_{\text {pre, } I}$ value of the "equivalent" pre-stress into a cable or a strut of index $i$, considered as an external axial load acting at both extremities of a cable ( $>0$ because in traction) or a strut ( $<0$ because in compression)

$$
\begin{aligned}
\tilde{F}_{\text {pre }} & =\left(F_{\text {pre }, 1}, F_{\text {pre }, 2}, F_{\text {pre }, 3}, \ldots F_{\text {pre }, n_{c}+n_{s}}\right) \\
& =\left(t_{1}^{\text {pre }}, t_{2}^{\text {pre }}, t_{3}^{\text {pre }}, \ldots t_{n_{c}+n_{s}}^{\text {pre }}\right) * \beta_{\text {pre }} * F,
\end{aligned}
$$

with

$-1 \leq t_{i}^{\text {pre }} \leq 1$, defining the value of $F_{\text {pre }}$ in each of the $\left(n_{c}+n_{s}\right)$ elements

$H$ height of the structure before application of external load $\tilde{F}$

$i, j, k$ integers used for iterations $(=0,1,2, \ldots)$, or index of a node, of a strut or a cable

$I_{s}, I_{s, i}$ cross-sectional moment of inertia of a strut of index $i$

$L \quad$ span of the structure

$L_{\text {def }}$ length of an element (strut or cable) when the structure is subjected both to the pre-stress $\tilde{P}$ and the external load case $\tilde{F}$

$L_{i n i}, L_{i n i, i}$ theoretical length of an element (strut or cable) of index $i$ when the structure is not loaded and not pre-stressed (=initial distance between both nodes of an element)

$L_{0}, L_{0, i} \quad$ length of an element (strut or cable) of index $i$ when the structure is subjected only to the pre-stress $\tilde{P}$

$m$ integer number such as $\beta=k / m$

$n$ number of nodes

$n_{c} \quad$ number of cables

$n_{s} \quad$ number of struts

$N, N_{i}$ axial force in an element of index $i$ (strut or cable), considered positive if in traction, after application of the external load $\tilde{F}$

$N_{c, i}$ axial force in a cable of index $i$, considered positive if in traction (always positive), after application of the external load $\tilde{F}$

$N_{c, \max }$ maximum value of axial force $N_{c, i}$

$N_{c, \text { min }}$ minimum value of axial force $N_{c, i}$

$N_{s, j}$ axial force in a strut of index $j$, considered positive if in traction (always negative), after application of the external load $\tilde{F}$

$N_{s, \max }$ maximum value of the absolute value of axial force $N_{s, j}$

$\left(N_{k, X}, N_{k, Y}, N_{k, Z}\right)$ the components of internal force $N$ at the extremity "node $k$ " of an element (strut or cable), expressed in the $(X, Y, Z)$ system of axis 
$P, P_{I} \quad$ value of the pre-stress into a cable ( $>0$ because in traction) or a strut ( $<0$ because in compression) of index $i$, before application of the external load

$\tilde{p} \quad$ vector $\left(t_{1}^{P}, t_{2}^{P}, t_{3}^{P}, \ldots, t_{n_{c}+n_{s}}^{P}\right)$, with $-1 \leq t_{i}^{P} \leq 1$. It is the pre-stress state

$\tilde{P}=\left(P_{1}, P_{2}, P_{3}, \ldots, P_{n_{c}+n_{s}}\right)=\left(t_{1}^{P}, t_{2}^{P}, t_{3}^{P}, \ldots, t_{n_{c}+n_{s}}^{P}\right) * \beta * F$, with $-1 \leq t_{i}^{P} \leq 1$, defining the value of $P$ in each of the $\left(n_{c}+n_{s}\right)$ elements. It is the pre-stress scenario

$q$ defined only for struts as $q=I_{s} / A_{s}^{2}$

$Q_{i}$ axial force into an element of index $i$

$S$ number of elementary tensegrity modulus that compose the structure

$t_{i}^{F} \quad$ one value of vector $\tilde{f}$

$t_{i}^{P} \quad$ one value of the vector $\tilde{p}$

$t_{i}^{\text {pre }}$ one value of the vector $\tilde{f}_{\text {pre }}$

$u \quad \sigma_{c} / \sigma_{s}$

$V$ volume of materials (cables + struts)

$W$ indicator of volume, equal to $\sigma_{s} V / F L$

$(X, Y, Z)$ coordinates of the global system of axis
$\left(X_{i}, Y_{i}, Z_{i}\right)$ coordinates of node $i$ in the global system of axis, before application of external load $\tilde{F}$

$\alpha_{X}, \alpha_{Y}, \alpha_{Z} \quad$ see Figure 4

$\alpha_{d e f, X}, \alpha_{d e f, Y}, \alpha_{d e f, Z}$ see Figure 4

$\beta, \beta_{\text {pre }}$ factor $>0$ defining the pre-stress level

$\beta_{\text {min }}, \beta_{\text {premin }}$ value of $\beta$ or $\beta_{\text {pre }}$ under which the least tensioned cable slacks

$\delta$ particular value of $d_{i}$, for example, the deflection at mid-span

$\theta \quad$ real number $\geq 1$

$\lambda_{i}$ slenderness of a strut of index $i$, equal to $\mu L_{0} \sqrt{A_{s, i} / I_{s, i}}$

$\Lambda_{i}$ equal to $\lambda_{i} /\left(\pi \sqrt{E_{s} / \sigma_{s}}\right)$

$\rho$ volumic weight of a material

$\sigma$ can be $\sigma_{c}$ or $\sigma_{s}$

$\sigma_{c}$ maximum allowable stress in cables

$\sigma_{s}$ maximum allowable stress in struts

$\Psi$ buckling indicator of the structure, equal to $\Psi=\sigma_{s} L / \sqrt{q E F}$ 